\title{
Light-weight Trust-enhanced On-demand Multi-path Routing in Mobile Ad Hoc Networks
}

\author{
Hui Xia ${ }^{1,2,3^{*}}$, Jia Yu $^{1}$, Cheng-liang Tian ${ }^{1}$, Zhen-kuan Pan ${ }^{1,3}$, Edwin Sha ${ }^{4}$ \\ 1 College of Information Engineering, Qingdao University, Qingdao 266071, P.R.C \\ 2 Shandong Provincial Key Laboratory of Software Engineering, Shandong University, Ji'nan 250101, P.R.C \\ 3 Postdoctoral Research Station of System Science, Qingdao University, Qingdao 266071, P.R.C \\ 4 Department of Computer Science, University of Texas, Dallas 75083-0688, USA \\ Corresponding E-mail: xiahui@sdu.edu.cn
}

\begin{abstract}
Mobile ad hoc networks (MANETs) are originally designed for a cooperative environment, which are vulnerable to a wide variety of attacks due to their intrinsic characteristics. Trust can be introduced to address this security issue at some level. In this paper, we focus on the concept of trust and abstract a decentralized trust inference model, where the trust an entity has for a neighbor forms the basic building block of this model. Basing on the interest entity's historical behaviors, multi-dimensional trust attributes are incorporated to reflect trust relationship's complexity in various angles. The weight vector of attributes is calculated by fuzzy AHP scheme based on entropy weight measure. The trust inference framework provides the considerable security with an additional small overhead, which can be incorporated into any routing protocol. In this paper, the standard Ad hoc On-demand Multi-path Distance Vector protocol (AOMDV) is extended as the base routing protocol to evaluate this model. The proposed light-weight trust-enhanced routing protocol (TeAOMDV) can provide a feasible approach to choose an optimal two-way trusted route without containing the untrustworthy entities instead of the shortest route, thus mitigate the impairment effects from such entities. It is light-weight in the sense that the trust framework uses only passive and local monitoring information to evaluate the behaviors of an interest entity which is translated to an estimate of the trust, consumes limited computational resource. Moreover, the new proposed data-driven route maintenance mechanism reduces routing overhead and route discovery frequency. The simulations show that the proposed routing scheme behaves better in attack resistance (i.e., grey-hole attack and black-hole attack), and makes an improvement on the packets delivery ratio, routing packets overhead, route discovery frequency and malicious node detection. Finally, as an extension of the trust model, by utilizing the trust assessment data sequence, we propose an improved SCGM(1,1)-Markov chain prediction method based on the system cloud grey model and Markov stochastic chain theory to forecast entity's trust level for future decision making.
\end{abstract}

Index Terms: decentralized trust inference model; trust attributes; fuzzy AHP; light-weight trust-enhanced routing protocol; data-driven route maintenance mechanism; attack resistance; malicious node detection

\section{Introduction}

In the last decade, researchers have explored many potential applications of mobile ad hoc networks (MANETs), which are usually deployed in harsh or uncontrolled environments. Due to the intrinsic characteristics (e.g., wireless medium, openness, the absence of a fixed infrastructure), such networks are vulnerable to a wide variety of attacks. Routing is a very important function in MANETs, which is productive unless all component nodes operate by a trustworthy manner. However, this is not always the case. The protocols used in this function are designed for minimizing the level of overhead and allowing every node to participate in the routing process, while usually not designed with security in mind and often are very vulnerable to node misbehaviors. Moreover, making routing protocols efficient often increases the security risk of the protocol and allows a single node to significantly impact the operation of the protocol because of the lack of protocol redundancy. An adversary or a malicious node can easily launch attacks on this important function, especially attacks on the packet routing (e.g., grey-hole, black-hole, cheating, or modification attack). The normal communications in network may be prohibited or hindered due to these attacks. The distribution of false routing information may cause the potential of denial of service attacks, unintended network routing loops, or other nonfunctional routes. Therefore, the security of routing protocol is an important area that needs to be addressed for such networks to widespread adoption.

To address the above issues, a variety of security-considered routing protocols have been proposed. The motivation of designing these protocols is that the networks need to determine the validity and safety of routing information prior to making routing decisions [1]. Basing on the protection way of reducing or eliminating malicious attacks, these protocols can be classified into two types, secure routing protocol and trust-based routing protocol. Most of secure routing protocols use cryptographic primitive techniques to secure the routing information. However, the disadvantage of using cryptographic tools is that the computational resource is very expensive, which is not suitable to be used in the resource-constrained mobile devices. These protocols create new avenues for denial of service (DoS) attacks in the attempt to prevent some other attacks [2,3]. Besides, several such secure routing protocols presume the existence of a centralized or distributed trusted third party, while this party actually violates the nature of self-organization [3]. Moreover, such protocols usually cannot against or prevent the malicious attacks from malicious or compromised nodes which have been authorized as the participants in network from doing misbehaviors. Thus, it is becoming more acceptable to consider trust-based routing as a viable security solution. Such protocols attempt to establish trusted routes rather than shortest routes as is done in traditional routing protocols, which make tradeoff between the trustworthiness and the performance of networks. The establishment of such protocols contains two important segments, trust model and trust-enhanced routing strategy. Every node in the network independently executes a designed trust model. The task of trust model is to 
periodically quantify and establish the trustworthiness among entities based on some trust metrics or trust computational methods [1 12].The estimated trust value can be directly applied to ensure proper operations, such as routing decision, authentication, access control, malicious node detection, and intrusion detection system [13] (e.g., basing on the trust feedback information obtained from the trust inference model, the network participants could be reminded to participate in the network transaction with caution, the selection of next hops or forwarding paths). However, introducing the 'trust' will increase much excessive overhead. Therefore, the design principle for trust-based routing protocol in MANETs should be simple, effective, and with a low communication cost.

In this paper, we focus on the security of routing protocol in MANETs. Firstly, we abstract a decentralized trust inference model. Then by extending the standard Ad hoc On-demand Multi-path Distance Vector protocol (AOMDV), we propose a novel light-weight trust-enhanced routing protocol combined with the trust model, named as TeAOMDV. The persuasive experiments have been conducted to simulate and present the effectiveness of this new protocol.

The main technical contributions of our work are summarized as follows:

1. We firstly give the definition and derivation of trust, then abstract a decentralized trust inference model, where the trust an entity has for an interest neighbor forms the basic building block of this model. Basing on the interest entity's historical behaviors, multi-dimensional trust attributes are incorporated to reflect trust relationship's complexity in various angles. The weight vector of attributes is calculated by fuzzy AHP scheme based on entropy weight measure.

2. The standard Ad hoc On-demand Multi-path Distance Vector protocol (AOMDV) is extended as the base routing protocol to evaluate the proposed trust model. In the trust-enhanced routing strategy, Hop Count, Forward Path Trust and Reverse Path Trust, the three metrics compose a three-dimensional evaluation vector for routing decision which provides a flexible and feasible approach to establish multiple two-way trusted paths without containing the untrustworthy entities instead of the shortest route.

3. This new protocol (TeAOMDV) is light-weight, containing three layers of meaning: (1) the intrusion detection system (IDS) used for estimating the trust that one entity has for another, consumes limited computational resource; (2) the trust framework uses only passive and local monitoring information to evaluate the behaviors of an interest entity which is translated to an estimate of the trust; (3) the new proposed data-driven route maintenance mechanism, termed as path trust alert mechanism, reduces routing overhead and route discovery frequency.

4. The simulations show that the proposed routing scheme behaves better in attack resistance and makes an improvement on the packets delivery ratio, routing packets overhead, route discovery frequency and malicious node detection. There is a tradeoff between route optimality and route credibility. Moreover, at the same time, the simulation results prove that the recommendation mechanism does play an important role in trust assessment.

5. As an extension of our trust model, by utilizing the trust assessment data sequence, we propose an improved SCGM(1,1)-Markov chain prediction method based on the system cloud grey model and Markov stochastic chain theory to forecast entity's trust level for future decision making.

The remaining paper is organized as follows. Section 2 discusses the literature work. In Section 3, we describe our decentralized trust inference model in detail. Basing on the proposed trust framework, in Section 4, we propose a novel light-weight trust-enhanced routing protocol, named as TeAOMDV. Section 5 presents the experiments and analysis on the performance of the new protocol. We make a detailed analysis of our model in Section 6. As an extension of our trust model, we propose an improved SCGM(1,1)-Markov chain prediction method in Section 7. Finally, Section 8 gives the concluding remarks along with extensions and directions for future research.

\section{Related Work}

Over the last few years, a number of trust-based schemes, trust-based systems and trust-based applications have been developed. As we are planning on adopting trust in MANETs, we firstly focus our attention on some trust models, and subsequently do a deep research on the modifications of routing strategy proposed in trust-based routing protocols.

\subsection{Trust Models in MANETs}

By analyzing the existing trust models, we can see that although a lot work has been done on the trust quantification, the research on trust is far from enough. A detailed survey on various trust computing approaches was presented [1]. The summary and comparisons of these approaches were highlighted. The following content are some efficient schemes used for estimating trust in MANETs found in the different references.

Observing a node's historical behaviors (e.g., packets delivery ratio, fully cooperation, bandwidth, residual energy and CPU usage) is an effective measure which is used to judge whether an interest entity is credible or not. In order to compute an interest entity's trust, we can introduce some polymerization mechanisms to aggregate these observations. The authors in [2] argued that in general, this trust problem could be viewed as an instance of detecting nodes whose behavior was an outlier when compared with others. The search, evaluation, propagation, and evolution among each entity are combined to provide an independent trust evaluation model for a MANET [3], trust was determined only by self-relevant knowledge.

At present, most of the trust evaluation frameworks belong to a recommendation-based methodology such that the evaluation results are heavily dependent on the accurate measurement of the behaviors of neighboring entities and on the degree of honesty of the raters [3]. Most researchers are advocating the use of recommendation and prefer making use of recommendation aggregation algorithms to quantify 'trust' from several aspects. The authors [4] proposed a judgement that whether the recommendation plays the same positive effect in the open computing environment as that used in human society. Then the persuasive experiments shown the problems existed in such mechanisms are that the recommendation is not always as helpful as what we expected, especially when the system is facing highly dynamic peer behaviors and 
malicious raters. Besides, such mechanisms put too much time and energy on the design of a complex recommendation aggregation measure, at the same time also increase the load of network entities. In paper [5], a monitoring entity may get recommendation experience by trust propagation along multiple directed recommending paths. By using the trust attenuation rules and the recommending path weighting rules proposed in this paper, this monitor can calculate these recommending paths' general weights and get the final recommendation.

Velloso et al. proposed a human-based model which described a maturity-based trust relationship between entities for MANETs in [6]. The trust-based scheme aimed to isolate or discourage selfish behaviors of participating nodes. In [7], the authors sought to combine the notions of 'social trust' derived from social networks with 'quality-of-service (QoS) trust' derived from information and communication networks to obtain a composite trust metric. Then they proposed an improved trust model in paper [8]. In this new model their interest is not so much in isolating selfish nodes but in quantifying the tradeoff between individual and global welfare, allowing each node to adapt to network dynamics and node status. Security in ubiquitous environment is a field that is growing up fast and spans across several fields. The authors [9] proposed a novel approach that establishes trust leveraging users' profiles. The information enables a task-aware trust model, namely a finer-grained model in which users are classified as trusted or not depending on the intended business activity. A recommendation subsystem based on the Wilson score confidence interval was introduced for the purpose of enabling the system to recognize malicious users. Two fundamental parameters (trust value and reputation value) were used in [10]. The nodes with highest trust values are used to build the headship induction table and the high value of reputation of a node signifies that the node is trusted and is more reliable for data communication purposes.

In order to forecast trust for future decision making, Onolaja et al. [11] proposed a reliable and novel dynamic framework that utilized a data-driven approach for trust management. The framework used past interactions, recent and anticipated future trust values of every identity in the domain. In order to reduce the hazards from such nodes and enhance the security of network, the authors present a dynamic trust prediction model to evaluate the trustworthiness of nodes, which is based on the nodes' historical behaviors, as well as the future behaviors via extending the fuzzy logic rules prediction method [12].

\subsection{Trust-based Routing Protocols in MANETs}

Applying trust mechanisms to routing strategy in MANETs is an intensive research field, and numerous solutions have been proposed. The selection of next hop or forwarding path is made according to the trust feedback information.

A light-weight trust-based routing protocol was presented in [14]. With the benefits of consuming limited computational resource and ensuring scalability, the intrusion detection system (IDS) and the local information were used to estimate the trust. They integrated the proposed trust approach into the standard AODV and gave the performance analysis. In paper [15], by utilizing the friendship measure, the authors proposed a trust-based scheme for securing AODV routing protocol. Before forwarding the data through the candidate routes, the sending nodes can evaluate the available routing paths according to some selected features (e.g., identity information or node reputation). The authors applied a simple trust approach into the source routing mechanism [12]. The novel on-demand trust-based unicast routing protocol provides a flexible and feasible approach to choose the shortest route that meets the security requirement of data packets transmission. Then they made some improvements on the former trust model [5], and proposed an on-demand trust-based multi-path routing protocol (AOTMDV) [13], in which two novel trust mechanisms were proposed. Firstly, the Message Authentication Code (MAC) mechanism was used to protect the new fields added in the routing control packets. Secondly, in order to cope with the frequent changes of route trust, the route trust update mechanism was proposed. One of the main contributions in paper [3] was to enable a feasible delivery method for defending against possible selfish and malicious behaviors rather than just securing routing protocol messages. Furthermore, the authors proposed a new routing framework in a MANET based on a sophisticated trust model.

The authors proposed a new mechanism termed as ASHFIK [10], which was used to make the routing protocol capable to maintain the group communications. In this mechanism, a new trusted core node is always ready to replace the original core node when it goes down. Cho and Chen proposed and analyzed a trust management protocol [8] for group communication systems where selfish nodes exist and system survivability is highly critical to mission execution. Rather than always encouraging altruistic behaviors, they considered the tradeoff between a node's individual welfare vs. global welfare. Moreover, in order to balance selfish vs. altruistic behaviors, they also identified the best design condition of this behavior model. The authors in [16] designed a routing solution that enables the dynamic source routing protocol to find a secure end-to-end route free of black hole nodes with the cooperation from the neighbors. Also this solution can protect the network in the presence of colluding attackers without the need of promiscuous monitoring the neighbor nodes.

\section{Decentralized Trust Inference Model}

Mobile ad hoc networks are more susceptible to the destruction of malicious attacks or denial of cooperation, due to their physical characteristics. Quantifying trust in such a network is a challenging task. In this section, we present a technique that is used for estimating entity's trust. For the purpose of minimizing the overhead in a resource-constrained environment as that of a MANET, we have used only passive monitoring to evaluate the behaviors of a node. Subsequently, the behaviors are translated to an estimate of trust, which the monitoring entity has on the monitored one [5].

\subsection{Trust Definition and Derivation}

The notion of trust used throughout this paper is defined [17] as: trust quantified by one entity is the degree of belief about the future behaviors of its interest entities. Trust can also be defined as the service provider not only follows the requesting entity's willingness, but also has the capability to deliver a mutually agreed service in a given context. 
In order to accurately quantify 'trust', coping with the subjectivity and uncertainty contained in its definition, we need a flexible and adjustable model. The subjectivity of trust comes mainly from the entity's own direct experience (e.g., historical observations, related actions). By taking advantage of other entities' experience or information, recommendation information can be used to alleviate the influence from the monitoring node's subjective manner. The two main reasons that the uncertainty existed in trust definition are, the bad surrounding environment and the sudden change of the interest entity's behaviors. For example, a MANET environment may contain unpredictable obstacles, such as mountains, lakes or buildings, impeding or blocking message relay. The obstacles not only restrict the nodes movement but may obstruct the effective transmission paths between nodes. We can solve the uncertainty problem of trust by monitoring the change of physical environment and assessing the degree of entity activity.

In conclusion, before quantifying the trust of an interesting entity, we firstly need to define trust key attributes, which are the computing indicators. These key attributes should well reflect the behavior characteristics of the entity, as to make the trust computation objective and efficient. In this paper, multi-dimensional trust attributes, including direct trust, recommendation trust, forward delivery ratio, historical trust record and activity degree, are incorporated to reflect trust relationship's complexity in various angles.

\subsection{Trust Model Assumption}

We make some assumptions before designing the trust inference framework: (1) In order to ensure the practicality of trust model, we follow the tenet that the 'trust' should be defined and quantified locally. In other words, the trust value is quantified only using the local information for scalability [14]; (2) The communications between two physical neighbors (one-hop) are considered more reliable than those of multi-hop communications; (3) For the purpose of identifying misbehaving nodes, each monitoring node should be equipped with some local detection mechanism [12] (e.g., in this paper, the neighbors' behaviors can be monitored via using the promiscuous mode).; (4) The wireless link is symmetrical, while the 'trust' is not necessarily symmetric between two physical neighborhood entities; (5) The cooperative action in the network interaction is encouraged, which is naturally required in such networks.

Basing on the above assumptions, a mobile ad hoc network with $n$ nodes can be abstracted. Due to the mobile nodes join, leave, or fail over time, the number of $n$ may be dynamically changing. In such networks, trust is a relationship between any two physical neighbor entities, which also can be described as an edge of a directed graph abstracted from the graph theory $[12,17]$. Under permitting conditions, each node in the trust system is initially authenticated by an authentication method. In our trust model, every node maintains a trust value for each of its neighbors. This value is a measure of the credible degree of low and high, defined in a continuous range between 0 and 1 (i.e., $0 \leq T V_{i j} \leq 1$ ). Let $v_{i}$ and $v_{j}$ denote the monitoring node and the monitored node, respectively.

\subsection{Trust Assessment}

There has been substantial work on the trust frameworks, which is divided into two types, the decentralized approaches and the centralized approaches. The former approaches are precise and do not suffer from single point of failure, they are not global in nature and are biased. Thus in this section, we abstract a decentralized trust inference model to quantify the 'trust' for entities based on multi-dimensional trust attributes.

\subsubsection{Model Construction}

Assume the set of multi-dimensional trust attributes is $\mathbf{A}=\left\{a_{j} \mid j=1,2, \ldots, n\right\}$, the attribute weight vector is $\mathbf{W}=\left\{w_{1}, w_{2}, \ldots\right.$, $\left.w_{n}\right\}$, and $w_{j} \geq 0, \sum_{j=1}^{n} w_{j}=1$. At time $t$, the characteristic vector of multi-dimensional trust attributes is $c(t)=\left\{c_{1}, c_{2}, \ldots, c_{n}\right\}$. The trust of the monitored entity can be quantified by utilizing the aggregation of the characteristic vector, described as:

$$
T V(t)=\sum_{j=1}^{n} w_{j} c_{j}
$$

\subsubsection{Trust Attributes Selection}

In this paper, five trust attributes are selected to quantify the 'trust' for an interest entity.

\section{A Direct Trust}

The calculation of this trust attribute is mainly utilizing the monitor's own direct experience (e.g., historical observations, related actions). We firstly introduce two definitions,

Definition 1 Correctly Forwarding Ratio $(C F R)$ : The proportion of the number of correctly forwarding packets to the number of packets supposed to be forwarded [12]. Correctly forwarding means that a forwarding node not only transmits a packet to the intended node but also forwards devotedly. If the sender monitors that one neighbor carries out an illegal modification, the $C F R$ of this neighbor will decrease.

Definition 2 Window Correctly Forwarding Ratio $(C F R(t))$ : The window correctly forwarding ratio $C F R(t)$ is the packet forwarding ratio in a recent window $W . C F R(t)$ is computed as follows:

$$
C F R(t)=\left\{\begin{array}{cl}
\frac{N_{C}(t-W, t)}{N_{A}(t-W, t)}, & t>W \\
\frac{N_{C}(t)}{N_{A}(t)}, & t \leq W
\end{array}\right.
$$

where $N_{C}(t-W, t)$ represents the cumulative number of correctly forwarding packets and $N_{A}(t$ - $W, t)$ signifies the total number of all requesting packets during the recent window $W$. The history records out of the recent window are discarded. 
In order to detect whether a packet is successfully forwarded by the monitored node or not, the sender does not delete the packet immediately. The packet will be stored in the packet buffer [12]. If the sender in the promiscuous mode monitors that the packet is forwarded correctly, this packet will be removed from the packet buffer and the corresponding number of correct forwarding $\left(N_{C}(t-W, t)\right)$ is increased by 1 .

In any network systems, packets are classified into two types: the control packets and the data packets, and the control packets play a vital role in establishing routing paths. Thus in this paper, the $C F R$ is also divided into two types: the control packet correctly forwarding ratio, denoted by $C C F R$, and the data packet correctly forwarding ratio, denoted by $D C F R$. The above two types of packets forwarding ratio need to be assigned proper weights for the purpose of estimating the observations. At time $t$, the direct trust of entity $v_{j}$ can be evaluated by entity $v_{i}$ via the following equation:

$$
D T_{i j}(t)=\alpha \times C C F R_{i j}(t)+(1-\alpha) \times D C F R_{i j}(t)
$$

\section{B Recommendation Trust}

Similar to the human society, recommendation trust mechanism is an important component in the vast majority of trust systems. The core of recommendation mechanisms is designing the recommendation aggregation algorithms. Two adjacent entities may not be adjacent due to the node's mobility, while they may obtain each other's trust assessment information for a fixed period. The monitoring (or evaluating) entity needs to collect the recorded trust information from the trusted raters, in order to modify the calculations reasonably.

We hold the opinion that it is not worth putting too much time and energy on the design of a complex rating aggregation measure, especially in open computing environments for trust inference. We can obtain such similar persuasive effect via introducing a simplified rating model. The recommendation result is calculated as,

$$
R T_{k j}(t)=\frac{\sum_{k=0}^{n-1} D T_{i k}(t) \times D T_{k j}(t)}{\sum_{k=0}^{n-1} D T_{i k}(t)} D T_{i k}(t) \geq \text { Trust_Threshold }
$$

where $v_{i}$ is the monitoring entity, $v_{k}$ is the potential raters (within $l$-hop physical neighbors, the parameters $l$ is used to control the network overhead.), and $v_{j}$ is the monitored entity. $D T_{i k}\left(t_{i}\right) \geq$ Trust_Threshold indicates that the monitoring entity only admits the recommendations from the trusted raters.

\section{Forward Delivery Ratio}

The forward delivery ratio $(F D R)$ is the measured probability that a data packet successfully arrives at the recipient. This metric reflects the state of the physical link and surrounding environment. The lower of this metric, the bad of the surrounding environment that the entity exposures.

The delivery ratio is measured using dedicated link probe packets as described below. Each node broadcasts link probes of a fixed size, at an average period $\tau$ ( 0.2 second in the implementation). To avoid accidental synchronization, $\tau$ is jittered by up to $\pm 0.1 \tau$ per probe. Because the probes are broadcast, $802.11 \mathrm{~b}$ does not acknowledge or retransmit them. Every node remembers the probes it receives during the last $w$ seconds, allowing it to calculate the delivery ratio from the sender at any time $t$ as:

$$
F D R_{i j}(t)=\frac{N(t-w, t)}{w / \tau}
$$

where $N(t-w, t)$ is the number of probes received during the window $w$, and $w / \tau$ is the number of probes that should have received. In the case of the link $v_{i}{ }^{-}>v_{j}$, this technique allows $v_{j}$ to measure $F D R$. Because $v_{j}$ knows it should receive a probe from $v_{i}$ every $\tau$ seconds, $v_{j}$ can correctly calculate the current loss ratio even if no probes arrive from $v_{i}$. Due to the assumption that the wireless link is symmetrical, thus we can calculate this metric via node $v_{i}$.

\section{Historical Trust Record}

In order to assess an accurate trust value of an interest entity, we need to refer this entity's historical trust record. For example, a MANET may face the cheating attacks. In order to cheat a high reputation, a malicious entity may make good performance (or disguise itself to be a good one) in a special time interval, and then behave badly. If we only focus on the entity's behaviors of the current interval, this malicious entity will be mistaken for a trusted one. In order to eliminate such things, the historical trust record should be considered in trust calculation.

\section{$E$ Activity Degree}

Activity degree reflects the level of activity and stability of entities in the network. Either the more number of trusted raters or the higher of activity level indicates that the entity has a higher credibility. If a monitored entity has a higher activity degree, other entities are willing to interact with it due to its expected higher trust level.

$$
A D_{i j}(t)=\frac{1}{2}\left[\phi(L)+\phi\left(n_{\text {total }}\right)\right] \quad \phi(x)=1-(1 / x+\delta)
$$

where $L$ represents the cumulative number of trusted raters for the monitored entity, $n_{\text {total }}$ represents the cumulative number of entities interacting with the entity. $\delta$ is an arbitrary constant greater than zero, used to control the speed of $\phi(x)$ value tend to 1 . The bigger of $\delta$, the higher speed tend to 1 .

\subsubsection{Weight Calculation for Multi-dimensional Trust Attributes}

In the process of quantifying trust for an interest entity, how to determine the weight of multi-dimensional trust attributes is critical. Though the weight information of trust attributes is partially known, to give precise weight vector is very difficult. In this section, we introduce the fuzzy AHP scheme based on entropy weight measure [17] to determine the weight of multi-dimensional trust attributes. The decision-maker can specify preference expression about the importance 
of each attribute. The system combines these preferences using fuzzy AHP [18] to re-emphasize attribute priorities. In the fuzzy-AHP procedure, the pairwise comparisons in the judgment matrix are fuzzy numbers that are modified by the decision-maker's emphasis. Using fuzzy arithmetic and $\alpha$-cuts, the procedure calculates a sequence of weight vectors that will be used to combine the scores on each attribute. The procedure calculates a corresponding set of scores and determines one composite score that is the average of these fuzzy scores.

The specific computational process is divided into the following six steps:

Step 1: Assume the number of multi-dimensional trust attributes is $n$, and the number of feasible solutions for weight calculation is $m$. In order to indicate the relative strength of the relevant trust attributes in the hierarchy judgment matrix, the symmetric triangular fuzzy number set $\left\{\begin{array}{lllll}\tilde{1} & \tilde{3} & \tilde{5} & \tilde{7} & \tilde{9}\end{array}\right\}$ can be used to compare the performance score. The traditional comparison matrix used in AHP method can be deformed into the fuzzy comparison matrix $\tilde{X}$, in which the fuzzy comparison vector for trust attribute $a_{i}$ is $\left\{\tilde{x}_{1 i}, \tilde{x}_{2 i}, \ldots, \tilde{x}_{m i}\right\}^{T}$ :

$$
\tilde{X}=\left[\begin{array}{ccc}
\tilde{x}_{11} & \cdots & \tilde{x}_{1 n} \\
\vdots & \ddots & \vdots \\
\tilde{x}_{m 1} & \cdots & \tilde{x}_{m n}
\end{array}\right]
$$

Step 2: We multiply the fuzzy subjective weight vector $\tilde{W}_{0}$ with the corresponding column of fuzzy comparison matrix $\tilde{X}$ for the purpose of building the fuzzy judgment matrix $\tilde{J}=\tilde{W}_{0} \times \tilde{X}$ :

$$
\tilde{J}=\left[\begin{array}{ccc}
\tilde{w}_{1} \times \tilde{x}_{11} & \cdots & \tilde{w}_{n} \times \tilde{x}_{1 n} \\
\vdots & \ddots & \vdots \\
\tilde{w}_{1} \times \tilde{x}_{m 1} & \cdots & \tilde{w}_{n} \times \tilde{x}_{m n}
\end{array}\right]
$$

Step 3: Except for the multiplication operation, the interval arithmetic $\alpha$-cuts is introduced. We should firstly define a triangular fuzzy number based on a triplet $\left(a_{1}, a_{2}, a_{3}\right)$. This function is shown in the following equation:

$$
\mu_{\hat{J}}(x)=\left\{\begin{array}{cc}
0, & x<a_{1} \\
\frac{x-a_{1}}{a_{2}-a}, & a_{1} \leq x \leq a_{2} \\
\frac{a_{3}-x}{a_{3}-a_{2}}, & a_{2} \leq x \leq a_{3} \\
0, & x>a_{3}
\end{array}\right.
$$

Alternatively, by defining the interval of confidence at level $\alpha$, we can characterize the triangular fuzzy number:

$$
\forall \alpha \in[0,1], \hat{J}_{\alpha}=\left[a_{1}^{\alpha}, a_{3}^{\alpha}\right]=\left[a_{1}+\left(a_{2}-a_{1}\right) \alpha, a_{3}-\left(a_{3}-a_{2}\right) \alpha\right]
$$

Basing on the equation $(8,9)$, the fuzzy judgment matrix $\tilde{J}$ can be deformed to:

$$
\hat{J}_{\alpha}=\left[\begin{array}{ccc}
{\left[a_{11 L}^{\alpha}, a_{11 R}^{\alpha}\right]} & \cdots & {\left[a_{1 n L}^{\alpha}, a_{1 n R}^{\alpha}\right]} \\
\vdots & \ddots & \vdots \\
{\left[a_{m 1 L}^{\alpha}, a_{m 1 R}^{\alpha}\right]} & \cdots & {\left[a_{m n L}^{\alpha}, a_{m n R}^{\alpha}\right]}
\end{array}\right]
$$

where $a_{i j L}^{\alpha}=w_{i L}^{\alpha} \times x_{i j L}^{\alpha}, a_{i j R}^{\alpha}=w_{i R}^{\alpha} \times x_{i j R}^{\alpha} \quad(0<\alpha \leq 1$ and for all $i, j)$

Step 4: The degree of satisfaction of this judgment matrix $\hat{J}_{\alpha}$ can be estimated by using an optimism index $\lambda$, when the number $\alpha$ is fixed. The optimism index $\lambda$ indicates the degree of optimism of a decision maker, which is a linear convex combination defined as $\hat{a}_{i j}^{\alpha}=\lambda \hat{a}_{i j L}^{\alpha}+(1-\lambda) \hat{a}_{i j R}^{\alpha}, \forall \lambda \in[0,1]$. A larger $\lambda$ indicates a higher degree of optimism. In the following experiments, we set $\alpha=0.8, \lambda=0.5$. The parameter settings denote a moderate decision maker. The matrix $\hat{J}_{\alpha}$ can be further converted into the following matrix $\hat{A}$ :

$$
\hat{A}=\left[\begin{array}{ccc}
\hat{a}_{11}^{\alpha} & \cdots & \hat{a}_{1 n}^{\alpha} \\
\cdots & \ddots & \cdots \\
\hat{a}_{m 1}^{\alpha} & \cdots & \hat{a}_{m n}^{\alpha}
\end{array}\right]
$$

Step 5: In a $n$ decision factors with $m$ feasible solutions problem, basing on the total fuzzy judgement matrix $\hat{A}$, the entropy of $j$-th decision factor (trust attribute) can be calculated by the following equation:

$$
H_{j}=-K \sum_{i=1}^{m} f_{i j} \log _{2} f_{i j}(i=1,2, \ldots, m ; j=1,2, \ldots, n)
$$


In which $f_{i j}=r_{i j} / \sum_{i=1}^{m} r_{i j}, r_{i j}=\frac{\hat{a}_{i j}^{\alpha}-\min \left\{\hat{a}_{i j}^{\alpha}\right\}}{\max _{j}\left\{\hat{a}_{i j}^{\alpha}\right\}-\min _{j}\left\{\hat{a}_{i j}^{\alpha}\right\}}, K$ is a constant $K=\frac{1}{\ln m}$.

Step 6: Finally, the weight of the $j$-th trust attribute can be calculated based on the information entropy measure:

$$
w_{j}=\frac{1-H_{j}}{n-\sum_{j=1}^{n} H_{j}}, 0 \leq w_{j} \leq 1, \sum_{j=1}^{n} w_{j}=1
$$

\subsection{Trust Table}

We use the time stamp mechanism to analyze each interaction interval, corresponding with the time window $W$. Till to the current time $t$, there are $t / W$ interaction intervals between two entities (i.e., $\mathbf{T}=\left\{t_{i} \mid i=1,2, \ldots, n\right\}$ ). Based on section 3.3 , we can get a trust vector of an interest entity $\mathbf{T V}=\left\{T V\left(t_{1}\right), T V\left(t_{2}\right), \ldots, T V\left(t_{n}\right)\right\}$. We focus on the last value of this vector, which will be written in the trust table of each node (i.e., Table 1).

Table 1 Node $\mathrm{v}_{i}$ 's trust table

\begin{tabular}{|c|c|c|}
\hline Neighbor_ID( $\left.\boldsymbol{v}_{\boldsymbol{i}}\right)$ & $\boldsymbol{T V}$ & Black-List $\left(\boldsymbol{v}_{\boldsymbol{i}}\right)$ \\
\hline$v_{k}$ & 0.908 & 0 \\
\hline$v_{m}$ & 0.779 & 0 \\
\hline$v_{j}$ & 0.232 & 1 \\
\hline$\ldots$ & $\ldots$ & $\ldots$ \\
\hline
\end{tabular}

In the view of the monitoring entity, if the trust value of an interest neighbor is smaller than the malicious threshold (e.g., $\eta 0.35$ set in the experiments), this neighbor will be classified as a malicious node contained in the malicious-list field of trust table. If an entity is regarded as the malicious entity by all its neighbors, it will be excluded from the local network.

\section{Trust-enhanced On-demand Multi-path Routing Protocol}

Our proposed trust inference framework can be incorporated into any routing protocol. As an application, a novel trust-enhanced on-demand multi-path routing protocol is proposed (termed as TeAOMDV), using the standard AOMDV as the base routing protocol. Any mobile node in this trust system has the ability to quantify the trust for each neighbor and select the trustworthy path to transmit data stream.

\subsection{Routing Table}

Definition 3 Path Trust: this vector indicates the credible degree for transmitting data stream using the potential routing path, which can be used as a constraint in path discovery. In other words, when a sender desires to transmit data stream to any receiver, it needs to discover a routing path and assess its credibility.

In this study, the path trust is calculated according to the reliability of each node on this path. The rationale is that as soon as any node is untrustworthy, the entire path is untrustworthy. Due to the asymmetry of 'trust', this vector can be divided into two types, the forward path trust and the reverse path trust. The former is along the direction of data flow, and the latter is the reverse direction. Specifically speaking, the former represents the subjective judgment of the source, which is used to make a decision whether or not to transmit the data stream by this path. And the later represents the subjective judgment of the destination, whether or not to receive the data stream from this path.

$$
\text { PathTrust }_{s d}=\left\{\begin{array}{r}
\text { ForwardPathTrust }=\min \left\{\begin{array}{c}
T V_{m k} \\
s \leq m \leq d-1 \\
k=m+1
\end{array}\right. \\
\text { ReversePathTrust }=\min \left\{\begin{array}{c}
T V_{k m} \\
s \leq m \leq d-1 \\
k=m+1
\end{array}\right.
\end{array}\right\}
$$

where $v_{s}$ is the sender, $v_{d}$ is the receiver, $v_{m}$ and $v_{k}$ are any two adjacent nodes on the candidate routing path, and $v_{m}{ }^{-}>v_{k}$ denotes that $v_{k}$ is the next hop of $v_{m}$.

\begin{tabular}{|c|}
\hline Destination \\
\hline Sequence Number \\
\hline Advertised Hop Count \\
\hline Expiration Timeout \\
\hline 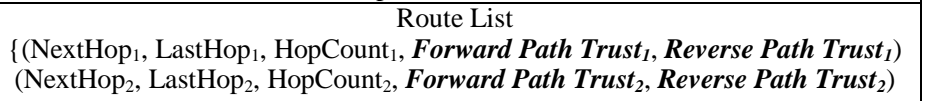 \\
\hline
\end{tabular}

Table 2 Routing entries of TeAOMDV

According to the above description, two new fields (i.e., Forward Path Trust (FPT) and Reverse Path Trust (RPT)) are added into the original routing entries of AOMDV, shown in Table 2. Hop Count, Forward Path Trust and Reverse Path Trust, the three metrics compose a three-dimensional evaluation vector for routing decision, which provides a flexible and feasible approach to establish multiple two-way trusted paths. 


\subsection{Route Discovery}

The source launches a route discovery process when there are no qualified routes existed in its routing table. Firstly, the source initiates a network-wide flood by broadcasting a route request packet and waits for route reply packets. Two new fields are added into RREQ packet, i.e., Reverse Path Trust (RPT) and Required Trust (RT). The value of field RPT is calculated based on the minimum of the continued product of trust that the RREQ packet has passed on this path, which is initialized to 1 and varies with the packet transmission. We introduce $R T$ to represent the path trust requirement, which remaining unchanged during this control packet flooding.

$R R E Q$ forwarding strategy: After an intermediate node $\mathrm{v}_{j}$ receives an $R R E Q$ packet from a neighbor node $v_{k}$,

Step 1: If node $\mathrm{v}_{j}$ has no route to this neighbor node $v_{k}$, it will create a route entry with the filed Reverse Path Trust $\left(R P T_{k j}\right)=T V_{j k}$ in its local routing table.

Step 2: Then it will check whether a same RREQ has been received or not. If so, assume both of the two packets satisfy the trust requirement, while the later copy has no less Hop Counter or greater sequence number, then the $R R E Q$ will be discarded and the process ends; otherwise, go to the next step. On the contrary, if the packets fail to meet the trust requirement, they will not be forwarded and deleted immediately. Any intermediate node may receive multiple $R R E Q$ copies from other intermediate nodes, then go to step 2.

Step 3: If node $v_{k}$ is not the source, node $v_{j}$ creates a reverse route to the source using the previous hop (node $v_{k}$ ) of the $R R E Q$ as its next hop $[6,20]$. The value of filed $R P T$ is set to $\min \left[R P T_{s k}, T V_{j k}\right]$ when $T V_{j k}$ is known, otherwise the value is $\min \left[R P T_{s k}\right.$, Trust_Threshold].

Step 4: If node $v_{j}$ has a valid route to the destination in its routing table, and the route's SequenceNumber is greater than the DestSequenceNo in the RREQ, node $v_{j}$ will generate an $R R E P$ to node $v_{k}$. Otherwise, node $v_{j}$ modifies the $R P T$ of the $R R E Q$ using $\min \left[R P T_{s k}, T V_{j k}\right]$ when $T V_{j k}$ is known, or min [RPT $T_{s k}$, Trust_Threshold] when $T V_{j k}$ is unknown [6, 20]. Then node $v_{j}$ increases the value of field Hop Count by one and propagates this modified $R R E Q$ packet to all neighbors.

Three new fields are also added into the packet RREP packet. The field Forward Path Trust (FPT) represents the minimum of the continued product of trust that the RREP has passed in route reply, which is initialized to 1 . The new added field Required Trust $(R T)$ has the same meaning as that in the RREQ. And the field Reverse Path Trust (RPT) is set to min [RPT(obtained in $R R E Q), R P T\left(P a t h_{j d}\right)$ ]. If an intermediate node has a fresh route to the destination and the received $R R E Q$ packet has not been processed previously, this node will send a route reply $(R R E P)$ packet via reversing back the path of $R R E Q$. If the destination receives multiple copies of $R R E Q$, it will reply the first $k$-paths at most. The parameter $k$ is used to control the number of RREPS and prevent an RREP storm [20]. If an intermediate node receives an $R R E P$, it will send the RREP via unicast unless the Forward Path Trust of the route which the RREP has passed is less than the Required Trust. When the RREP travels back to the source via traversing the path of the RREQ, each node on this path finally can set up a trusted forwarding route.

\subsection{Route Maintenance}

The route maintenance mechanism in this new protocol is similar with the standard AOMDV. A node maintains and updates routing entries in its own routing table when receiving a routing control packet (i.e., route request (RREQ), route reply $(R R E P)$, route error $(R E R R)$, and path trust alert (PTA) packet).

\subsubsection{Path Trust Alert Mechanism}

In this study, we propose a novel data-driven route maintenance mechanism, termed as Path Trust Alert. We convert the route error packet into the path trust alert packet by adding a new field flag $P$ in the reserved field after field $N$, shown in Table 3. The value of Flag $P$ set to 1 indicates that the packet is a path trust alert packet. When the path trust is lower than the trust requirement, a path trust alert event will be triggered. The path id field could distinguish the different paths via using the last hop field in its own routing table entries.

Table 3 PTA packet of TeAOMDV

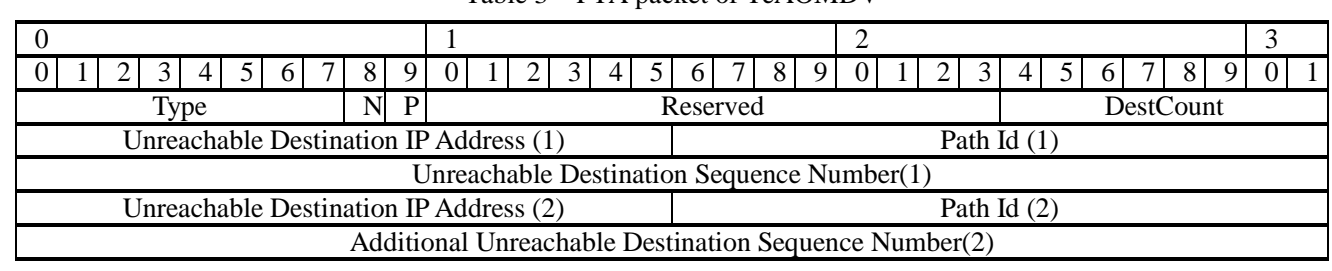

When an intermediate node receives a data packet, it will select its next hop according to the routing entry. And at the same time, it will also check whether its potential next hop's trust is greater than the trust threshold or not. If not, which indicates that the next hop is not a trustworthy node (i.e., suspect or malicious node) and a path trust alert event will be triggered. In this case, a PTA packet will be sent to its previous hop with the help of precursor list in routing table via unicast.

After an immediate node receiving a path trust alert packet PTA, it should traverse the unreachable destination list of $P T A$ and check whether the previous hop of the corresponding single path (which reach unreachable destination) in routing table is the previous hop of PTA. If such type of path exists, the relevant paths must be deleted and the corresponding unreachable destination will be added to a new path trust alert packet whose next hop is set to the previous hop of the concerned path. After traversing the unreachable destination list, they will be sent via unicast. Until the source node receives a PTA, it will delete the corresponding routing path and choose an optimal candidate one. The rationale is that, 
due to the link-disjoint routing mechanism, the trust change of any node on the forwarding path has nothing to do with the other candidate paths.

The ultimate goal of the mechanism is in response to the sudden or hidden malicious nodes in the routing path, in order to maintain the efficiency of routing. Compared with other trust route maintenance mechanisms [5, 12], this new proposed on-demand data-driven mechanism could reduce the routing overhead and route discovery frequency. The main reasons are: (1) This new mechanism is more suitable to the 'trust' criterion, destination, path id rather than only destination; (2) the previous hop of each path in the precursor list is used to control the propagation range of the route error message. Two simple cases for this new mechanism will be shown in the following subsection 4.4.2.

\subsubsection{Loop Freedom}

On-demand protocols in MANETs may encounter routing loops problem due to that they discover routes with the help of broadcasting mechanism. Sequence number mechanism effectively guarantees loop freedom. That is, for the purpose of avoiding the possibility of any cycle, each node maintains an increasing serial number. Destination sequence number are tagged on all routing packets, so as to provide a mechanism to calculate two relatively fresh routing packets generated two different nodes of the same destination.

An immediate node creates a reverse path to the source only when receiving a fresh control packet $R R E Q$, and a forwarding path to the destination with the RREP. At some time, an immediate node $v_{j}$ receives a control packet to a destination $d\left(v_{j} \neq d\right)$ from a neighbor $v_{k}$. The variables SeqNumber ${ }_{k}^{d}$, HopCounter ${ }_{k}^{d}$ and ReversePathTrust ${ }_{k}^{d}$ represent the DestSequenceNo, HopCounter and ReversePathTrust of the control packet respectively. Let SeqNumber ${ }_{j}^{d}$, RouteList ${ }_{j}^{d}$, MaxTrust ${ }_{j}^{d}$ and MinHops ${ }_{j}^{d}$ be SequenceNumber, RouteList, maximum PathTrust and minimum HopCount of multiple paths to destination $d$ in the routing table of node $v_{j}$ respectively. Combined with $R R E Q$, the update rule for route entries in routing table is shown as follows:

1. if $\left(\right.$ SeqNumber $_{j}^{d}<$ SeqNumber $\left._{k}{ }^{d}\right)$ then //a fresher $R R E Q$ packet

2. $\quad$ SeqNumber ${ }_{j}^{d}=$ SeqNumber $_{k}^{d}$;

3. $\quad$ RouteList ${ }_{j}^{d}=\mathrm{NULL}$;

4. if $\left(T V_{j k}\right.$ is unknown) then NewReversePathTrust=min[ReversePathTrust ${ }_{k}{ }^{d}$, Trust_Threshold];

5. else if $T V_{j k}>$ Trust_Threshold, NewReversePathTrust=min[ReversePathTrust $\left.{ }_{k}^{d}, \overline{T V}_{j k}\right]$;

6. $\quad$ insert $\left(k\right.$, HopCounter ${ }_{k}+1$, NewReversePathTrust $)$ into RouteList ${ }_{j}^{d}$;

7. $\quad$ node $v_{j}$ rebroadcasts the $R R E Q$;

8. $\quad$ else if $T V_{j k}<$ Trust_Threshold;

9. $\quad$ node $v_{j}$ discards this packet;

10. end if

11. else if $\left(\right.$ SeqNumber $_{j}{ }^{d}=$ SeqNumber $\left._{k}{ }^{d}\right)$ then

12. if ( $\min \left[\right.$ ReversePathTrust $\left.{ }_{k}{ }^{d}, T V_{j k}\right]>$ PathTrust_Threshold) and $\left(\right.$ HopCounter $_{k}{ }^{d}<$ MinHops $\left._{j}{ }^{d}-1\right)$ then

13. if $\left(T V_{j k}\right.$ is unknown) then NewReversePathTrust=min(ReversePathTrust ${ }_{k}^{d}$, Trust_Threshold);

14. $\quad$ else NewReversePathTrust $=\min \left[\right.$ ReversePathTrust ${ }_{k}^{d}, T V_{j k}$;

15. endif

16. insert ( $k$, Hop Counter $_{k}+1$, NewReversePathTrust) into RouteList $_{j}$; $_{\text {; }}$;

$17 . \quad$ node $v_{j}$ rebroadcasts the $R R E Q$;

18. else node $v_{j}$ discards this packet;

19. endif

20. endif

As mentioned above, line 1, 4, 8, 11, 12 and 18 of the rule ensures loop freedom. The proposed protocol is only allowed to accept an alternate route with smaller hop count in accordance with meeting the trust requirement.

\subsection{Routing Example}

\subsubsection{A Simple Route Discovery Case}

A simple route discovery case is shown in Figure 1. The sequence numbers of all nodes are equal to 5 and the BroadcastID (or Request ID) of node $A$ is equal to 8 [5].

Node $A$ broadcasts an RREQ carrying two additional trust fields: Required Trust $(R T=0.7)$ and Reverse Path Trust $(R P T=1)$. When node $B$ receiving the $R R E Q$ : Firstly, it creates a reverse route to node $A$; Then if $T V_{B A}$ satisfying the trust requirement, node $B$ rebroadcasts a modified $R R E Q$ to all its neighbors. In the $R R E Q$ transmitted by node $B$, the field of Reverse Path Trust is set equal to $R P T_{A B}$ or $T V_{B A}$, i.e., $R P T_{A B}=0.86$. As shown in Figure $1(a)$, node $B$ may also receive another copy of the RREQ from node $F$. After node $B$ receiving the latter one, it creates reverse routes to node $F$ and node $A$, respectively. Then it will discard this packet due to that this packet carrying with a bigger hop count. When this happens, the routing table after node $B$ receiving RREQs is shown in Figure $1(b)$.

Similar to the above situation, node $F$ may also successively receives two $R R E Q s$ from node $B$ and node $E$, respectively [20]. There will be the following two cases: (1) If node $F$ receives the $R R E Q$ from node $B$ earlier (The routing entries in node $B$ will be changed, the second entry shown in Figure $1(b)$ will not be created), it will rebroadcast this $R R E Q$, carried with the value of filed HopCounter is 2 and Reverse Path Trust is $\min \left[R P T_{A B}, T V_{F B}\right]$ (i.e., $\min [0.86,0.8]$ ). When the second copy of $R R E Q$ arriving, node $F$ will discard it due to that the latter one does not have a smaller hop counter or a bigger sequence number compared with the former one; (2) If the $R R E Q$ from node $E$ arriving earlier, node $F$ will rebroadcast the $R R E Q$ to node $B$ and node $G$. As shown in this figure, $R R E Q$ traversals of the two paths have the same hop count under the premise of the two reverse paths both satisfying the trust requirement. Corresponding with our route 
discovery strategy, the latter copy of $R R E Q$ from node $B$ will be discarded. Both of the above cases may happen in theory. For simplification, in the following discussion and the experiment tests, we assume the latter one happens.

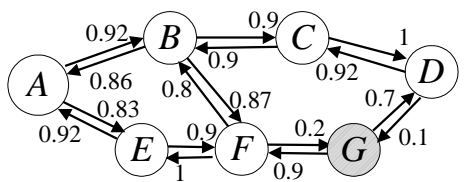

(a) Node Trust Values

\begin{tabular}{|c|c|c|c|c|c|c|c|}
\hline \multirow{2}{*}{ Dst } & \multirow{2}{*}{ SN } & \multirow{2}{*}{ ET } & \multicolumn{6}{|c|}{ Route List } \\
\cline { 4 - 8 } & & & NH & HC & LH & $\boldsymbol{F P T}$ & $\boldsymbol{R P T}$ \\
\hline$C$ & 5 & 6 & $C$ & 1 & $X$ & 1 & 0.92 \\
\hline$G$ & 5 & 7 & $G$ & 1 & $X$ & 1 & 0.1 \\
\hline$A$ & 5 & 8 & $C$ & 3 & $B$ & 1 & 0.86 \\
\hline$A$ & 5 & 8 & $G$ & 3 & $E$ & 1 & 0.1 \\
\hline \multicolumn{7}{|c|}{ (c) Routing table of node $D$} & Delete
\end{tabular}

after receiving $R R E Q \mathrm{~s}$

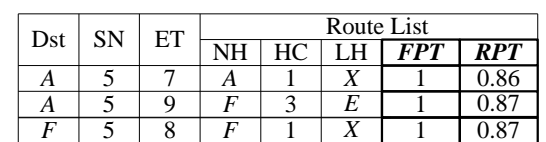

(b) Routing table of node $B$ after receiving $R R E Q \mathrm{~s}$

\begin{tabular}{|c|c|c|c|c|c|c|c|}
\hline \multirow{2}{*}{ Dst } & \multirow{2}{*}{ SN } & \multirow{2}{*}{ ET } & \multicolumn{6}{|c|}{ Route List } \\
\cline { 4 - 8 } & & & NH & HC & LH & $\boldsymbol{F P T}$ & $\boldsymbol{R P T}$ \\
\hline$A$ & 5 & 5 & $A$ & 1 & $X$ & 0.85 & 0.86 \\
\hline$A$ & 5 & 9 & $F$ & 3 & $E$ & 0.8 & 0.87 \\
\hline$F$ & 5 & 7 & $F$ & 1 & $X$ & 0.8 & 0.87 \\
\hline$C$ & 5 & 8 & $C$ & 1 & $X$ & 0.9 & 0.9 \\
\hline$D$ & 5 & 9 & $C$ & 2 & $C$ & 0.9 & 0.9 \\
\hline
\end{tabular}

(d) Routing table of node $B$ after

receiving only one RREP from node $D$
Dst: Destination

SN: Dst. Sequence Number

ET: Expiration Timeout

NH: NextHop

HC: HopCoun

LH: LastHop

FPT: Forward Path Trust

RPT: Reverse Path Trust

Note: The direction of the path from source to destination represents the forwarding direction

\begin{tabular}{|c|c|c|c|c|c|c|c|}
\hline \multirow{2}{*}{ Dst } & \multirow{2}{*}{ SN } & \multirow{2}{*}{ ET } & \multicolumn{6}{|c|}{ Route List } \\
\cline { 4 - 8 } & & NH & HC & LH & FPT & RPT \\
\hline$B$ & 5 & 7 & $B$ & 1 & $X$ & 0.92 & 0.86 \\
\hline$D$ & 5 & 9 & $B$ & 3 & $C$ & 0.9 & 0.86 \\
\hline
\end{tabular}

(e) Routing table of node $A$

after receiving $R R E P$ from node $D$

Figure 1. Route discovery instance

Following the above assumption, one copy of the $R R E Q$ will be forwarded by node $C$, and another copy will be forwarded by node $G$. After the former copy arriving at the destination node $D$, this node will create routes to node $C$ and node $A$, respectively. At the same time, it will send an RREP packet to node $C$. After one unit of time, the second copy from node $G$ arriving at node $D$, and then this node will create routes to node $G$ and node $A$, respectively. While the reverse path trust of $P(A, E, F, G, D)$ is equal to 0.1 and it is smaller than the trust requirement 0.7 . Therefore, node $D$ will discard this packet and will not create an RREP to node $G$. Corresponding with this case, the routing table of node $D$ is shown in Fig. 1(c). When node $B$ receiving an RREP from node $C$, it will create routes to node $C$ and node $D$ respectively in its own routing table. We can see from Fig. $1(d)$ that, the route entry to node $D$ is $<$ NextHop=C, HopCount=2, $F P T_{B D}=0.9, R P T_{B D}=0.9>$. At last, node $A$ will receive an $R R E P$ from node $B$, and create forwarding routes to node $B$ and node $D$. The routing table of node $B$ is show in Fig. 2(e), the finally decision routing path is the second route entry (named $r_{1}$ ), with the fields $<$ NextHop $=B$, Hop Count $=3, F P T_{A D}=0.9, R P T_{A D}=0.86>$.

Basing on the new route discovery strategy, node $A$ will find one route $P(A, B, C, D)$. Moreover, the malicious node $G$ is excluded in the route setup phase.

\subsubsection{Path Trust Alert Mechanism Cases}

If a node desires to sent/forward data stream while its next hop suddenly changed to a malicious node (e.g., captured by an adversary), this node will launch a path trust alert (PTA) event. Step by step, all the corresponding nodes will get the PTA message. When they have data to be sent, they will not select these paths containing malicious nodes.

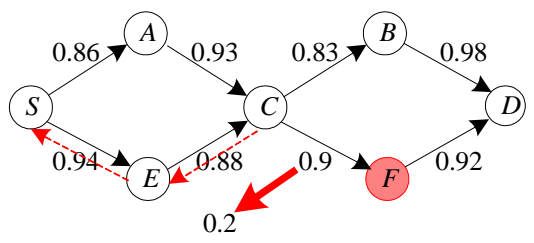

Figure 2. Route maintenance for the same pair source and destination

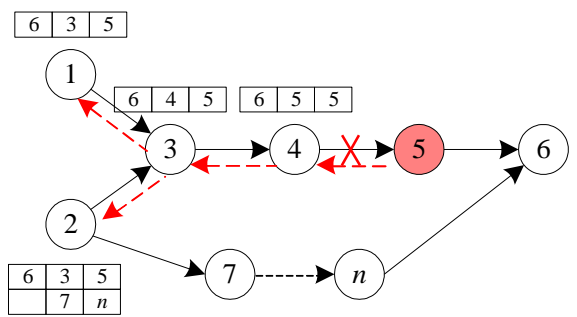

Figure 3. Route maintenance for the pair with same destination and different sources

The Path Trust Alert process can be simply demonstrated through Figure 2 and 3. In Figure 2, we assume that the value of field Trust_Threshold is 0.7, and the reverse path trust of both paths meets the trust requirement. Based on the new routing strategy, the choosing path is $P_{1}(S, E, C, F, D)$, and $P_{2}(S, A, C, B, D)$ is the second option. At some time, node $F$ is about to make malicious attack (e.g., with dropping $80 \%$ packets received from node $C$ ), and then the using path is about to be un-trusted. The node $C$ will detect this malicious behavior soon via utilizing the trust model proposed in Section 3 . When a data packet arrives at node $C$, node $C$ will launch a Path Trust Alert event, the last hop of the old routing path $P_{1}(S$, $E, C, F, D)$ is node $F$, thus the path id field of this original PTA packet will be $F$. Then node $C$ will send the $P T A$ to node $E$ via unicast step by step until the source node $S$ gets the message. After that, when a new data packet is to be sent, node $S$ will select the path $P_{2}(S, A, C, B, D)$ to replace $P_{1}$. If we continue to use the conventional route error mechanism of 
AOMDV, node $C$ will forward $R E R R$ to both node $E$ and node $A$, which will resulting in deleting all the two routing paths in the $S$ ' routing table. Therefore source node $S$ has to trigger a new route discovery process. As is demonstrated in this case, our strategy could propagate the PTA message along the reverse path and avoid rerouting discovery.

Figure 3 demonstrates another simple PTA case with the simplified routing table entry (i.e., destination node, next hop, last hop), both of nodes 1 and 2 are source nodes, and node 6 is the same destination. The routing path $P(3,4,5,6)$ is used for transmitting data packets by both of the two sources. At some time, node 5 is about to make malicious attack. After a short time, node 4 detects this malicious behavior, launches the path error event and unicasts the PTA packet to its upstream node 3 . The message is propagated to nodes 1 and 2 by the same way. Due to no other candidate routes, node 1 has to construct a new routing route via triggering a new route discovery process. And node 2 will switch its route to the candidate one (through by node 7) if it is available in this moment, as shown in Figure 3.

\section{Simulation Results and Analysis}

\subsection{Experiment Setup}

NS-2.35 [19] is adopted to evaluate the performance of trust-based protocols in different conditions. Our simulation models a network of 40 mobile hosts placed randomly within a $1000 \mathrm{~m} \times 1000 \mathrm{~m}$ area. Radio propagation range for each node is 250 meters and channel capacity is $2 \mathrm{M}$ bits/sec. Each simulation executes for 600 seconds of simulation time. We take an un-slotted Carrier Sense Multiple Access protocol with Collision Avoidance (CSMA/CA) to transmit data packets as well routing packets [20]. The IEEE 802.11b Distributed Coordination Function (DCF) is used as the medium access control protocol. A traffic generator is developed to simulate constant bit rate sources. The size of data payload is 512 bytes. The node mobility uses the random waypoint model. In the following tests, malicious nodes can launch two simple types of routing attacks, i.e., grey-hole and black-hole attacks. In the former one, malicious nodes selectively forward data packets at a ratio of 35, and in the latter one, malicious nodes drop all data packets, but both of them deliver route request and reply packets devotedly.

We simulate and compare the following schemes: the standard AOMDV, three deformation versions of AOMDV (AOTMDV [5], LTB-AOMDV [13] and new protocol TeAOMDV). TeAOMDV-1 denotes for that the trust attributes contains the rating, while not in TeAOMDV-2. Six metrics [6] are used to evaluate the performance of these mentioned routing protocols: (1) Packet delivery ratio: the fraction of the data packets delivered successfully at destination nodes to those sent by source nodes. This ratio represents the efficiency of routing; (2) Average end-to-end latency: the average time taken by the data packets from sources to destinations, including buffer delays during a route discovery, queuing delays at interface queues, retransmission delays at MAC layer and propagation time; (3) Routing packet overhead: the ratio of the number of control packets to the number of data packets. This metric is used to investigate how efficiently control packets are utilized in delivery data packets; (4) Path optimality: the proportion of the total number of hops in the optimal trusted path to the shortest hops number in theory; (5) Route discovery frequency: we define this metric by the number of route requests initiated by the source per second; (6) Detection ratio: the ratio of the number of nodes whose behavior (malicious) is identified correctly to the actual number of such nodes.

\subsection{Trust-enhanced Routing Evaluation}

To assess the performance of these protocols, we choose suitable parameters and simulate the following scenarios under various conditions as shown in Table 4.

Table 4 Varying simulation parameters

\begin{tabular}{|c|c|c|c|c|}
\hline Test Number & Malicious Node Number & Maximum Speed & Trust Threshold & Malicious Threshold \\
\hline 1 & 4 & $0-30 \mathrm{~m} / \mathrm{s}$ & 0.7 & 0.4 \\
\hline 2 & $0-10$ & $10 \mathrm{~m} / \mathrm{s}$ & 0.7 & 0.4 \\
\hline 3 & 10 & $10 \mathrm{~m} / \mathrm{s}$ & 0.7 & $0.1-0.5$ \\
\hline
\end{tabular}

Multiple runs with different seed numbers are conducted for each scenario. To decrease the disturbance of random error, every experiment repeats 30 times and the average experiment results are computed. These results are shown in Figure 4, Figure 5 and Figure 6.

\subsubsection{Test 1: Varying Node Maximum Speeds}

With nodes speeding up, Figure 4(a) illustrates that the delivery ratio of AOMDV declines remarkably while such ratios of the other three trust-enhanced protocols decrease gently. The faster the maximum speed, the more apparent illustrate the difference. Benefits from the high detection ratio of malicious node, the trust-enhanced protocols perform better. By making use of the 'trust' feature, such protocols elevate the probability of successful delivery. In contrast, the node in AOMDV only implements the traditional routing strategy (i.e., principle of shortest path first), which could not improve the packet delivery ratio when encountering intermediate malicious nodes. In other words, the traditional protocol is modified based on the trust enhancements so that the trusted route is chosen instead of the shortest route. TeAOMDV has a higher delivery ratio than the other two due to that the trust model proposed in TeAOMDV is more effective (e.g., trust computation with multi-dimensional trust attributes, solve the problem of subjectivity and uncertainty contained in trust).

As shown in Figure 4(b), the average end-to-end latency in these protocols rises with the increase of node maximum speed. The routing routes break down easily as the nodes speed up. The repair work brings a higher latency. The other three trust-enhanced protocols have lower average latency than AOMDV. The reason is that: the queuing delays and retransmission delays are two main reasons for causing the average end-to-end latency. Without considering the 'trust', 
there is a big probability of the malicious nodes appearing on the routing path. These nodes could launch modification, grey hole or black hole attacks, resulting in a much higher latency. While the data packets in trust-enhanced protocols may be forwarded along with a longer but trustworthy path. Comparing with AOTMDV and LTB-AOMDV, our new protocol has a better performance. Our new routing strategy in TeAOMDV is not searching for the most trusted path, but for a trusted routing path meeting trust requirement with the smallest hop count.

The routing packet overhead in those mentioned protocols changes as a function of node maximum mobility speed shown in Figure 4(c). Higher speeds lead to more route rediscoveries and more route maintenance, and consequently, trigger more control packets and higher overhead. The overhead in trust-enhanced protocols remains comparatively higher than that in AOMDV along with the speed increasing. The trust-enhanced protocols are the same as route break in AOMDV, so more route rediscoveries are required to launch for qualified routes to meet trust requirement in those protocols. In contrary, trust requirement is not considered in AOMDV. Our proposed protocol is better than the other two, the reasons are: (1) In route discovery, the modified $R R E Q$ packets transmission mode as described in subsection 4.2 , will reduce the number of $R R E Q$ and $R R E P$, so as to better control the routing overhead; (2) In routing maintenance phase, the new proposed path trust alert mechanism can also decrease the amount of control packets. Moreover, TeAOMDV-1 has a little bigger value than TeAOMDV-2, because of the trust model in the former one combining with the recommendation mechanism which increasing the routing overhead.

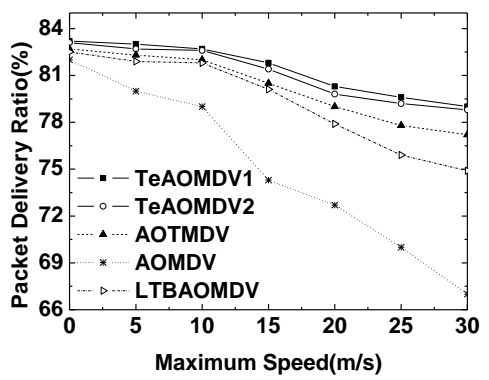

(a) Packet Delivery Ratio

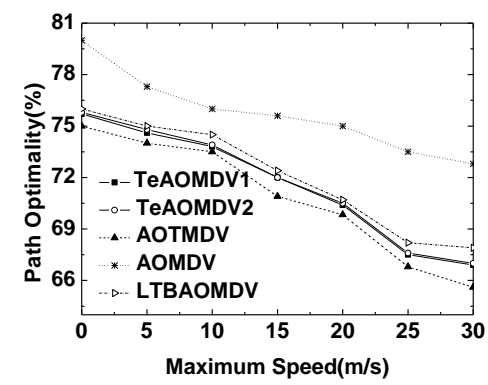

(d) Path Optimality

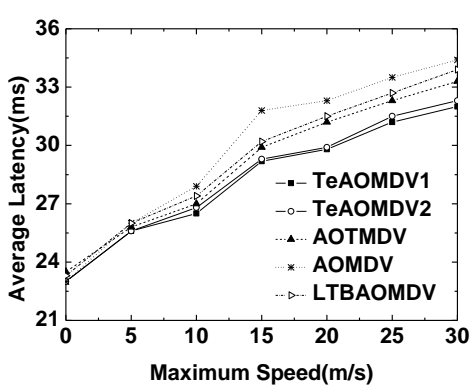

(b) Average End to End Latency

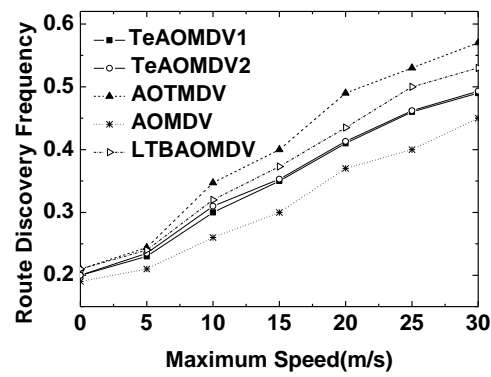

(f) Route Discovery Frequency

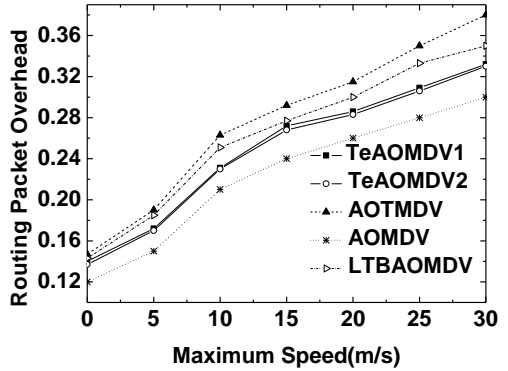

(c) Routing Overhead

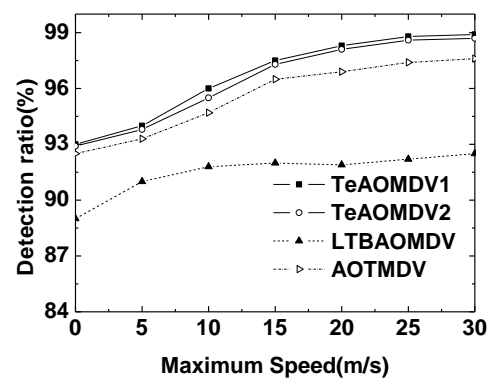

(e) Detection Ratio of Malicious Nodes

Figure 4. Test 1: Performance with varying node maximum speeds

We can see from Figure $4(d)$ that, the path optimality of these protocols degrades as the speed increases. AOTMDV, LTB-AOMDV and TeAOMDV have smaller path optimality than AOMDV. It is observed that the actual routes may not be the best available routes due to the 'trust' factor considering in trust-enhanced protocols, where source nodes make routing selection considering both the hop count and the path trust. Sometimes, only longer routes can satisfy trust requirement, which reduce the path optimality. Due to the route discovery strategy in TeAOMDV (i.e., choosing a trusted path with the smallest hop count), it performs better than the mechanism proposed in [5].

Figure 4(e) illustrates that the route discovery frequency in these four protocols rises with the increase of maximum speed. At higher speeds, routing paths become invalid more quickly. In order to find the potential routes, source nodes have to initiate more route rediscoveries, thus increases the number of route requests per second. Furthermore, for the purpose of finding a trusted route, AOTMDV, LTB-AOMDV and TeAOMDV further increase this mentioned number. TeAOMDV performs better than the other two trust-enhanced protocols. Because of that: (1) the trust model in TeAOMDV has better performance in estimating node's trust for future behaviors; (2) Once the path credibility is lower than the trust required, TeAOMDV triggers a PTA packet back to source node via uicast to delete the single invalid routing path rather than to delete all affected paths in route maintenance, which also can help network to reduce the route discovery frequency.

As shown in Figure 4(f), the malicious node detection ratio of AOTMDV, LTB-AOMDV and TeAOMDV descends with the increase of node maximum speed. We can observe that when the nodes move faster, the interactions among nodes increase gradually, and the trust information spreads faster and broader. This leads to a higher detection ratio of malicious nodes. Benefit from the multi-dimensional trust attributes, our new mechanism performs better than the other two.

\subsubsection{Test 2: Varying Number of Malicious Nodes}


In a normal network without any malicious node, the packet delivery ratio is approximately $93 \%$ in each protocol. This ratio of AOMDV degrades sharply as the number of malicious nodes increases from 0 to 10, as shown in Figure 5(a), while this indicator of TeAOMDV drops from $93 \%$ to $78 \%$ smoothly. The delivery ratios of AOTMDV, LTB-AOMDV and TeAOMDV are always much higher than that of AOMDV. The reason is that once the malicious nodes existed in routing path will cause serious lost package consequences. As an intelligent agent, any mobile node has the ability to quantify the trust for each neighbor and select the trustworthy path to transmit data stream, and therefore the delivery ratio is improved.

With the increasing number of malicious nodes, the average end to end latency in three trust-enhanced routing protocols ascends slowly while the average latency in AOMDV ascends sharply, as shown in Figure 5(b). The routing paths established by trust-enhanced mechanisms may add hops but can eliminate the influence of malicious nodes, leading to a longer delay. While in AOMDV, due to the lack of the consideration of the malicious nodes, such nodes inflict severe damage (e.g., grey-hole or black-hole attacks) on the routing functions. A large amount of data packets are needed to be re-forwarded, resulting in greater average end-to-end latency.

As shown in Figure 5(c), the routing packet overhead of trust-enhanced protocols is a little higher than that of AOMDV. The differences become more apparent at more malicious nodes. Regardless of the increase of malicious nodes, the overhead of AOMDV is around 0.2 and remains unchanged roughly, while inversely the overhead of trust-enhanced protocols ascends. The reasons are: (1) the increased control packets in trust-enhanced protocols are primarily due to their route discovery mechanism that sending more modified $R R E Q$ and $R R E P$ route packets to find trustworthy paths; (2) the increase of malicious nodes will result in more frequent path trust changes and route maintenance, leading to a higher overhead. Due to the proposed trust update mechanism, AOTMDV has the biggest value. Our new route discovery strategy and data-driven path trust alert mechanism could decrease the routing overhead.

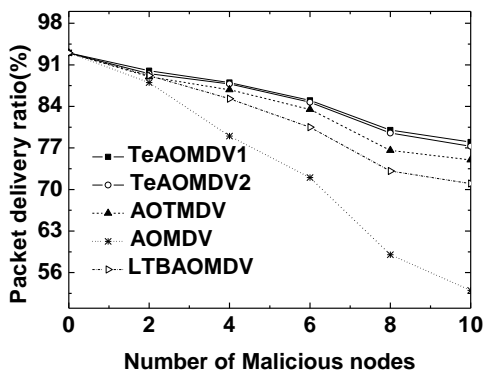

(a) Packet Delivery Ratio

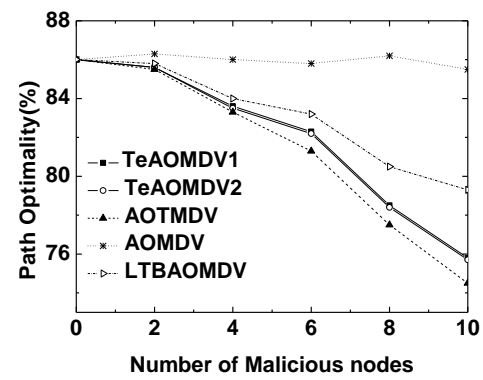

(d) Path Optimality

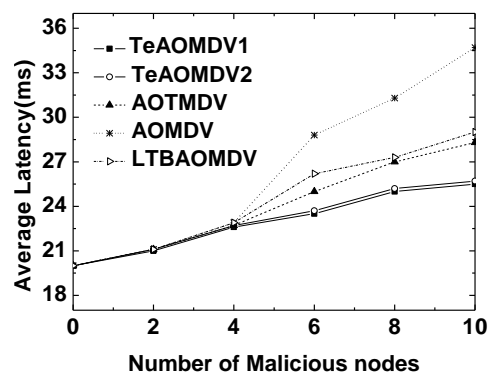

(b) Average End to End Latency

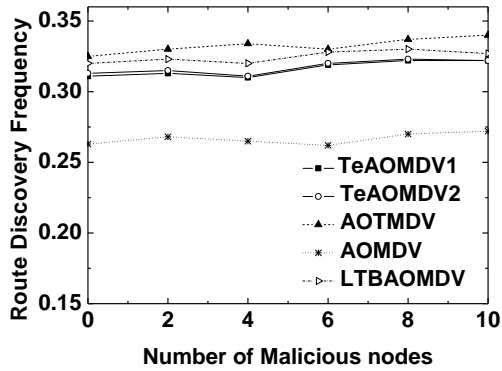

(e) Route Discovery Frequency

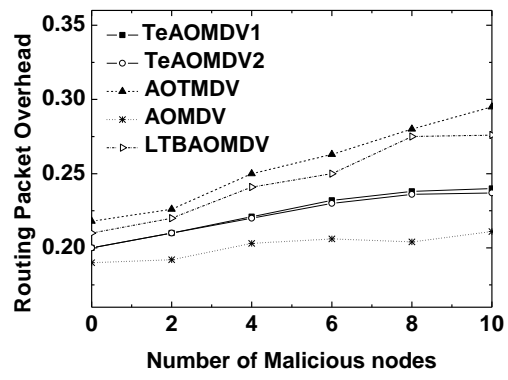

(c) Routing Overhead

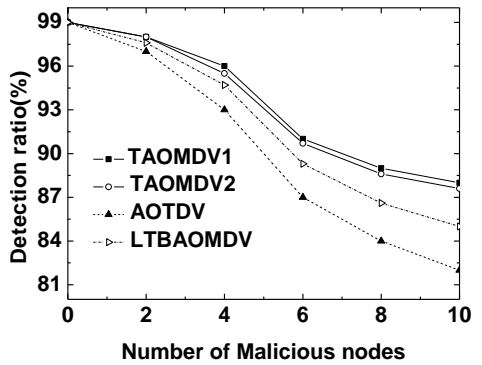

(f) Detection Ratio of Malicious Nodes

Figure 5. Test 2: Performance with a varying number of malicious nodes

Similar with Figure 4(d), AOMDV exhibits the best path optimality with the increase of the malicious nodes as shown in Figure $5(d)$. As malicious nodes increasing, the path optimality of AOTMDV, LTB-AOMDV and TeAOMDV decreases. AOMDV only implements the traditional routing protocol, which only maintains shorter routes to destinations, while the other three trust-enhanced routing protocols are able to detect and filter out malicious nodes. AOTMDV performs the worst; the reason is that this trust-enhanced protocol executes the routing strategy with choosing the most trusted path, which may find a longer routing path than the others. After modifying the route discovery strategy in AOTMDV, TeAOMDV has a better performance.

Figure 5(e) illustrates that the route discovery frequency in these four protocols remains unchanged roughly with the increase number of malicious nodes. And the AOMDV has the minimum value, because of that, it does not consider 'trust' factor in the route discovery strategy. Only when the link failure and no available candidate paths in the routing table entry, it will reroute discovery. TeAOMDV performs better than the other two trust-enhanced protocols; the reasons are the same as the illustration for Figure 4(e).

The detection ratio of AOTMDV, LTB-AOMDV and TeAOMDV is shown in Figure 5(f). They decline with the increase number of malicious nodes. It is obvious that the more malicious nodes are, the more serious their damage is, and the detection is harder. Benefit from the effective trust mechanism, the detection ratios are over $93.6 \%$ maintained in both types of TeAOMDV if the percentage of malicious nodes is not more than $25 \%$, and $4.3 \%$ higher than that of AOTMDV. 


\subsubsection{Test 3:Varying Malicious Threshold}

In this test, we compare TeAOMDV with AOTMDV by varying the malicious threshold from 0.1 to 0.5 . Ten nodes randomly selected in the network launch the above mentioned two kinds of packet routing attacks.

As shown in Figure 6(a), on the whole, the delivery ratios of TeAOMDV and AOTMDV are not as high as expected. In fact, they are all smaller than $80 \%$. This is because the proportion of malicious nodes is $25 \%$ and a lot of packets are forwarded by longer trusted routing path. Due to the transmission attenuation effect, the delivery ratio has an obvious attenuation. The delivery ratio of TeAOMDV increases slowly from $71 \%$ to $79.8 \%$ and then down to $77 \%$ with the malicious threshold increasing from 0.1 to 0.5 . A smaller trust threshold means that more packets could be dropped by a node before it is regarded as a malicious node. The delivery ratio of TeAOMDV-1, TeAOMDV-2 and AOTMDV reaches their peaks, respectively (i.e. $79.8 \%$ for TeAOMDV-1, 79.1\% for TeAOMDV-2 and 77.4\% for AOTMDV), when the threshold is equal to 0.4 . While this value is set to 0.5 , the delivery ratio has a dropping tendency. A higher malicious threshold results in a longer (hops) routing path, which increases the physical risk of packet transmission and causes a lower packet delivery ratio.

Figure 6(b) shows that the average end-to-end latency of TeAOMDV and AOTMDV changes as the malicious threshold ranges from 0.1 to 0.5 . And the average latency of TeAOMDV increases gradually from $21.2 \mathrm{~ms}$ to $27.3 \mathrm{~ms}$. When the malicious threshold is set to a bigger value, more nodes will be added to the black list, which leads to a longer routing path, higher average latency at the bigger threshold. The average latency of TeAOMDV-1 is very close to TeAOMDV-2, and both smaller than AOTMDV.

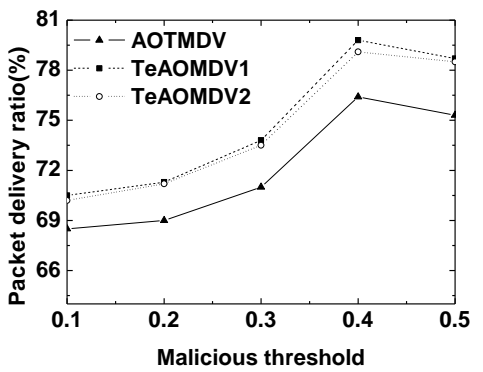

(a) Packet delivery ratio

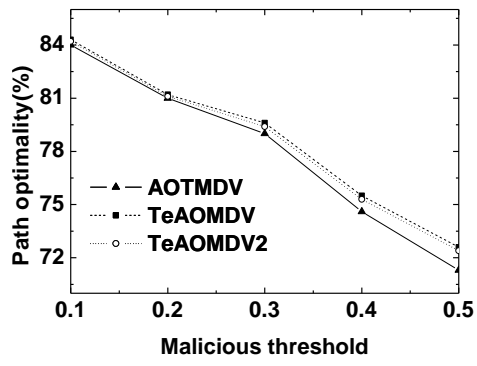

(d) Path optimality

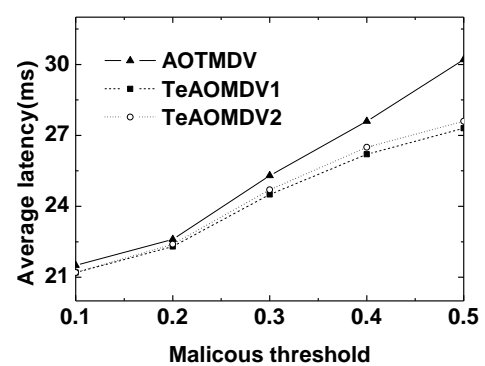

(b) Average end-to-end latency

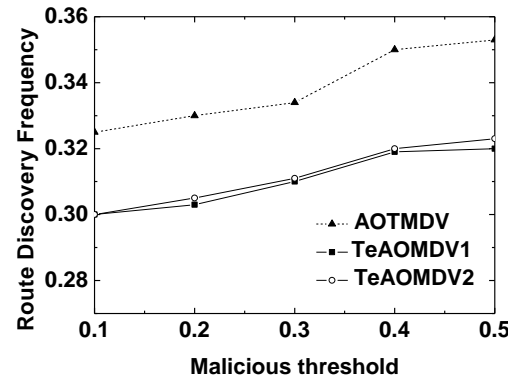

(e) Route discovery frequency

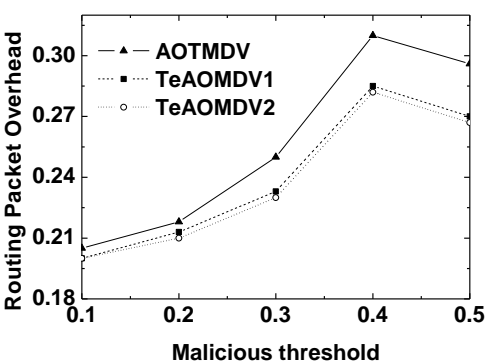

(c) Routing packet overhead

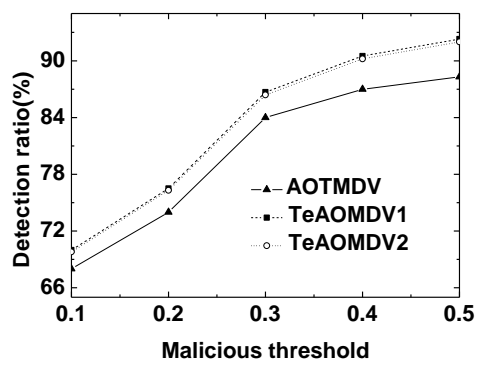

(f) Detection ratio of malicious nodes

Figure 6. Test 3: Performance with varying malicious threshold.

The effect on the routing packet overhead is shown in Figure 6(c). The average overhead of TeAOMDV is about 0.25 and this value of AOTMDV is about 0.27. If the malicious threshold is assigned to a small value (e.g., $\delta<0.35$ ), the malicious nodes launching grey-hole attack may not be detected and the count of route discoveries for avoiding malicious nodes is small. As the malicious threshold increases, more nodes are detected as un-trusted nodes and more routing packets are forwarded along more trustworthy and longer paths. Accordingly, the routing packet overhead tends to rise with the increase of the malicious threshold. With the help of the simple rating mechanism and light-weight route maintenance mechanism, the overhead in TeAOMDV is smaller than that in AOTMDV.

Figure $6(d)$ illustrates that the path optimality descends slowly with the increase of malicious threshold. The values of TeAOMDV and AOTMDV descend in the range from $84 \%$ to $72 \%$ as the malicious threshold increases. Overall, TeAOMDV and AOTMDV show similar performance in path optimality, and the TeAOMDV performs a little better.

The route discovery frequency ascends slowly with the increase of malicious threshold as shown in Figure 6(e). It's obvious that, bigger malicious threshold results in less candidate routes, which will cause more route rediscoveries when the used route is invalid. Benefiting from the improved trust model and path trust alert mechanism, the TeAOMDV performs a little better than AOTMDV.

As shown in Figure 6(f), the detection ratio of malicious nodes ascends with the increase of malicious threshold. The increase in the ratio of malicious nodes implies that when the trust threshold is set to a greater value, the malicious nodes are easier to be detected. As the malicious threshold rises to 0.5, TeAOMDV shows better performance in detection ratio about $4 \%$ than AOTMDV. 
The experimental results show that, TeAOMDV performs better than the other mentioned trust-enhanced protocols. There is a trade off between route optimality and route credibility. Moreover, TeAOMDV-1 shows a better performance than TeAOMDV-2, which indicates that the recommendation mechanism does play an important role in trust assessment.

\section{Trust Model Analysis}

\subsection{Trust Propagation and Mobility Affect}

By making use of the transitivity property of trust, the relevant and effective trust information can be propagated in the trust system. Basing on the trust inference model, any node in this trust system can identify the properties of its interest nodes (i.e., trustworthy node or not). And this trust information can be shared in the local scope via utilizing a higher layer. With the help of the node's mobility characteristic, the 'trust' information will be spread to the entire trust system (i.e., in technical parlance, they propagate trust information to other individuals). When the trust information propagated in the network, the resources spent on re-computation of trust can be reduced. The season is that, the trust information can be used to synthesize the trust on target, and alleviate the influence from evaluating node's subjective manners. The above description is particularly important in the network with the features of lack of infrastructure, wireless channel, mobility, open media, and resource-constraint.

The main factor of trust propagation is that the trust information should be propagated with nodes' full collaboration. If not every node, at least majority of the nodes should fully cooperate in transmitting relevant information. There are some cases to illustrate the concept of trust propagation, in which rating or recommendation mechanism is the simplest one. Under normal conditions, the rating is mostly from the direct physical one-hop neighbor especially can be of recommending paths (multi hops).

Basing on the concept of web of trust, a trust propagation approach for MANETs [21] is proposed, where multiple trusted nodes give rating experience about the unknown nodes. The evaluating node can modify the trust degree of other interest neighbors, based upon the rating experience. Then it will decide whether to accept the content from the concerned node or not.

By making use of node's mobility attribute, the propagation mechanism of trust information and security credentials is analyzed in [22]. There are certain policies considered, e.g., Friend nodes can carry the trust information and forward them as an authority device. When users meet, they are naturally given the possibility to visually identify each other. Basing on this physical encounter, setting up a security association between two nodes can be decided. In the trust system for mobile networks, the node's mobility attribute can make a great impact on the propagation of security credentials and trust information. The reason is that the nodes with higher mobility have more opportunity to interact with more new nodes.

\subsection{Against Attacks}

Trust computations can be attractive target for attackers since major decisions can be taken based on the trust results. In this study, we identify that two possible attacks (i.e., grey-hole and black-hole attacks) can be against by the new proposed trust scheme. In addition, this mechanism can be against several other kinds of attacks. (1) Since it is a deformation of neighbor sensing trust computing method, therefore it can handle the denial of service attacks, collusion attacks and hybrid approaches; (2) The mechanism can handle bad mouthing attack well because the rating is based on the aggregations of multiple observations; (3) In addition, there are big numbers of observers observing the target node, thus the new trust mechanism is also against the camouflage attacks well.

\section{Trust Model Extension}

Due to the inherent characteristics of MANETs, it is very hard for a monitoring node to assess whether the monitored node is trustworthy or not (e.g., willing to provide a requesting service or not) for the future time interval. If the trend can be forecasted accurately, the probability of risk occurrence can be eliminated. In a MANET, risk can be defined as poor transport state (e.g., false forward, bigger packet loss rate, link failure).

\subsection{Conventional Prediction Measures}

Some successful prediction measures already have been proposed (e.g., SVM theory, grey system theory, rough set theory). However, due to the complexity of trust concept, it is very hard to propose an effective model to predict a node's trust for the future time interval, especially combined with the incomplete trust assessment information and uncertainty small samples. In recent years, more and more researches have been focused on the combination of these conventional prediction measures. We adopt the system cloud grey model $\operatorname{SCGM}(1,1)$ to reveal the overall trend of prediction object in this paper. In addition, for the purpose of predicting the range of random fluctuation and optimize the results, we introduce the Markov stochastic chain theory which can enhance the preciseness and accuracy of data.

\subsection{SCGM(1, 1)-Markov Chain Prediction Model}

By making use of the obtained historical trust data sequence, we propose an improved SCGM(1,1)-Markov chain prediction model to effectively forecast an interest node's trust. We can obtain an accurate trust value for an interest node, which not only provides a relative identification between the normal nodes and the malicious nodes, but also offers a prediction for a node's future behaviors.

\subsubsection{SCGM(1,1) Construction}

For the convenience of description, the obtained trust vector $\mathbf{T V}=\left\{T V\left(t_{1}\right), T V\left(t_{2}\right), \ldots, T V\left(t_{n}\right)\right\}$ of an interest entity can 
be simplified to an original sequence of $\operatorname{SCGM}(1,1) \quad X^{(0)}=\left\{x^{(0)}(1), x^{(0)}(2), \ldots, x^{(0)}(n)\right\}$.

To suppress the influence of random observation errors, the averaged series are adopted,

$$
x^{(1)}(k)=\sum_{m=1}^{k} \bar{x}^{(0)}(m), \text { where } \bar{x}^{(0)}(k)=1 / 2\left(x^{(0)}(k)+x^{(0)}(k-1)\right)
$$

Then the original sequence $X^{(0)}$ can be changed to $X^{(1)}=\left\{x^{(1)}(2), x^{(1)}(3), \ldots, x^{(1)}(n)\right\}$.

A response function can be established according to the function $f_{r}(k)=b e^{a(k-1)}-c \quad\left(f_{r}\right.$ is defined as a certain function in time domain).

$$
a=\ln \frac{\sum_{k=3}^{n} x^{-(0)}(k-1) x^{-(0)}(k)}{\sum_{k=3}^{n}\left(x^{-(0)}(k-1)\right)^{2}} \quad b=\frac{(n-1) \sum_{k=2}^{n} e^{a(k-1)} x^{(1)}(k)-\sum_{k=2}^{n} e^{a(k-1)} \sum_{k=2}^{n} x^{(1)}(k)}{(n-1) \sum_{k=2}^{n} e^{2 a(k-1)}-\left(\sum_{k=2}^{n} e^{2 a(k-1)}\right)^{2}} \quad c=\frac{1}{(n-1)}\left[\left(\sum_{k=2}^{n} e^{a(k-1)}\right) b-\sum_{k=2}^{n} x^{(1)}(k)\right]
$$

Assume $x^{(1)}(k)=b-c, U=a c$, then the differential equation of $\operatorname{SCGM}(1,1)$ is $\frac{d \hat{x}^{(1)}(k+1)}{d k}=a \hat{x}^{(1)}(k+1)+U$. Then we can get the response function:

$$
\hat{x}^{(1)}(k+1)=\left(\hat{x}^{(1)}(1)+\frac{U}{a}\right) e^{a k}-\frac{U}{a}
$$

After reduction processing, we can get the final $\operatorname{SCGM}(1,1)$ prediction model:

$$
\hat{x}^{(0)}(k)=e^{a(k-1)} \frac{2 b\left(1-e^{-a}\right)}{1+e^{-a}}
$$

In equation (17), when $k=1,2, \ldots, n,\left\{\hat{x}^{(0)}(k)\right\}$ is the fitting sequence of $\left\{x^{(0)}(k)\right\}$; and $k>n,\left\{\hat{x}^{(0)}(k)\right\}$ is the prediction sequence of $\left\{x^{(0)}(k)\right\}$.

The relative error is:

$$
\varepsilon^{(0)}(k)=\left(x^{(0)}(k)-\hat{x}^{(0)}(k)\right) / x^{(0)}(k) \quad(k=0,1,2 \cdots)
$$

\subsubsection{Fuzzy Classification}

According to the relative error, the data sequence can be divided into $m$ states,

$$
\begin{aligned}
& \Theta_{i}=\left[\Theta_{i 1}, \Theta_{i 2}\right](i=1,2, \cdots, m) \\
& \Theta_{i 1}=\hat{x}^{(0)}(k) / 1-\delta_{i-1}, \Theta_{i 2}=\hat{x}^{(0)}(k) / 1-\delta_{i}
\end{aligned}
$$

where $\delta_{i-1}$ and $\delta_{i}$ are the lower boundary and upper boundary of relative error of the $i$ th state. $\Theta_{i 1}$ and $\Theta_{i 2}$ are actual values of the lower boundary and upper boundary of the $i$ th state. Because division standard is based on relative errors, the divided state zones of the system are not self-equal-width, and the width of each state zone would become broader when baseline ascends [23]. Given a relative error $x \in U$ ( $U$ is relative error set), its membership function can be established with triangle method as,

$$
u_{i}(x)=\left\{\begin{array}{cc}
1, & \delta_{0} \leq x \leq \frac{\delta_{0}+\delta_{1}}{2} \\
\frac{\delta_{1}+\delta_{2}-2 x}{\delta_{2}-\delta_{0}}, & \frac{\delta_{0}+\delta_{1}}{2} \leq x \leq \frac{\delta_{1}+\delta_{2}}{2} ; \\
0, & \text { else }
\end{array} u_{i}(x)=\left\{\begin{array}{cc}
\frac{2 x-\delta_{i-2}-\delta_{i-1}}{\delta_{i}-\delta_{i-2}}, & \frac{\delta_{i-2}+\delta_{i-1}}{2} \leq x \leq \frac{\delta_{i-1}+\delta_{i}}{2} \\
\frac{\delta_{i}+\delta_{i+1}-2 x}{\delta_{i+1}-\delta_{i-1}}, & \frac{\delta_{i-1}+\delta_{i}}{2} \leq x \leq \frac{\delta_{i}+\delta_{i+1}}{2} \\
0, & \text { else }
\end{array}\right.\right.
$$

Where $i=1, m$;

$$
u_{m}(x)=\left\{\begin{array}{cc}
\frac{2 x-\delta_{m-1}-\delta_{m-2}}{\delta_{m}-\delta_{m-2}}, & \frac{\delta_{m-2}+\delta_{m-1}}{2} \leq x \leq \frac{\delta_{m-1}+\delta_{m}}{2} \\
1, & \frac{\delta_{m-1}+\delta_{m}}{2} \leq x \leq \delta_{m} \\
0, & \text { else }
\end{array}\right.
$$

Therefore, the belongingness of each data can be described as a vector: $\left(u_{1}(x), u_{2}(x), \ldots, u_{m}(x)\right)$, named fuzzy state vector.

\subsubsection{Transition Probability Matrix Establishment}

Markov chain [24] refers to the random process $X(k)$, whose state only related to the state at the moment $t_{0}$ and nothing to do with the past states. The transition matrix that is also called the environment of Markov chain is a key factor of 
Markov chain.

Step 1. In Markov chain $\left\{x_{n}, n \in T\right\}$, let $P_{i j}=P\left\{x_{m+K}=j \mid x_{m}=i\right\},(i, j \in I)$ represents for the probability of the system changing its state from $i$ (at the moment $m$ ) to $j$ (at the moment $m+k$ ). Take $P_{i j}^{(k)}$ in order and construct the transition probability matrix:

$$
P^{(k)}=\left[\begin{array}{cccc}
P_{11}^{(k)} & P_{12}^{(k)} & \ldots & P_{1 \mathrm{n}}^{(k)} \\
P_{21}^{(k)} & P_{22}^{(k)} & \ldots & P_{2 \mathrm{n}}^{(k)} \\
\vdots & \vdots & \vdots & \vdots \\
P_{\mathrm{n} 1}^{(k)} & P_{\mathrm{n} 2}^{(k)} & \cdots & P_{\mathrm{nn}}^{(k)}
\end{array}\right]
$$

Any state in the system will be certainly transferred into one of listed states via $k$-steps in a row, so $\sum_{j=1}^{n} P_{i j}^{(k)}=1$. This matrix is also called $k$-steps transition probability matrix of Markov chain.

Step 2. Thus, corresponding with the above subsection, the probability of data sequence from state $\Theta_{i}$ to state $\Theta_{j}$ by $m$-steps transition is called $m$-steps transition probability and is recorded as:

$$
P_{i j}^{(k)}=\frac{m_{i j}^{(k)}}{M_{i}}
$$

where $m_{i j}^{(k)}$ is the number of state $\Theta_{i}$ transferred into state $\Theta_{j}$ by $m$-steps; and $M_{j}$ is the number of the appearance of state $\Theta_{j}$. However, when calculating the number of $M_{j}$, the last $k$ data must be deleted.

Step 3. In the terms of the distance from the to the prediction time, the transition steps are defined as 1-step, 2-steps,..., $j$-steps, respectively. Combining with the corresponding transition probability matrixes, the row vectors of the initial states are the probability of each state appearing, and then the sum of transition probability can be calculated [12].

\subsubsection{Error Correcting}

Step 1. Firstly, we define the symbol $F(\delta(n))$ as the vector of membership degree:

$$
F(\delta(n))=\left[u_{A 1}(\delta(n)), u_{A 2}(\delta(n)), \cdots, u_{A m}(\delta(n))\right]
$$

Where $\delta(n)$ is the relative error at times step $n$, and $u_{A 1}(\delta(n))$ is the membership degree of $\delta(n)$ in fuzzy state $A_{i}$.

Step 2. The vector of membership degrees at times step $n+1$ is still fuzzy vector, which can be calculated by the following formula:

$$
F(\delta(n+1))=F(\delta(n)) P=\left[u_{A 1}(\delta(n+1)), \cdots, u_{A m}(\delta(n+1))\right]
$$

Each component of $F(\delta(n+1))$ represents the membership degree of each fuzzy state at time step $n+1$. Taking the membership degrees in fuzzy vector as weights [25], the predicted relative error for the next step can be calculated by weight sum method:

$$
\delta(n+1)=\sum_{i=1}^{m} \frac{u_{A 1}(\delta(n))\left(\delta_{i-1}+\delta_{i}\right)}{2}
$$

Step 3. Lastly, the final value for the $n+1$ step can be calculated via the following formula,

$$
x^{(0)}(n+1)=\frac{\hat{x}^{(0)}(n+1)}{1-\delta^{(0)}(n+1)}
$$

\subsection{A Simple Node Trust Prediction Case}

We make an explanation for the new method via a simple case. Node's trust assessment values of different time series are used to construct patterns and clusters in the improved SCGM(1,1)-Markov chain prediction model. We can get the corresponding information listed in Table 5:

Table 5 The corresponding information of our new trust prediction model

\begin{tabular}{|c|c|c|c|c|c|c|}
\hline Seq. No. & Original Trust & Date Processing & SCGM(1,1) & Data Reduction & Relative Error \% & SCGM(1,1)-Markov Chain \\
\hline 1 & 0.991 & 6.8983 & 6.9117 & 0.998 & -1.1292 & 0.998 \\
\hline 2 & 0.862 & 6.7593 & 6.8053 & 0.903 & -4.7564 & 0.903 \\
\hline 3 & 0.633 & 6.4505 & 6.7011 & 0.814 & -28.5941 & 0.814 \\
\hline 4 & 0.644 & 6.4677 & 6.5986 & 0.735 & -14.1304 & 0.735 \\
\hline 5 & 0.734 & 6.5985 & 6.4977 & 0.664 & 9.5368 & \\
\hline 6 & 0.643 & 6.4661 & 6.3983 & 0.601 & 6.5319 & 0.664 \\
\hline 7 & 0.562 & 6.3315 & 6.3306 & 0.545 & 3.0249 & 0.601 \\
\hline 8 & 0.532 & 6.2766 & 6.2041 & 0.495 & 6.9549 & \\
\hline 9 & 0.440 & 6.0868 & 6.1091 & 0.450 & -2.2728 & 0.495 \\
\hline 10 & 0.420 & 6.0403 & 6.0156 & 0.410 & 2.3810 & \\
\hline 11 & 0.342 & 5.8348 & 5.9236 & 0.374 & -9.3567 & 0.450 \\
\hline 12 & 0.358 & 5.8805 & 5.8329 & 0.342 & 4.4693 & 0.374 \\
\hline 13 & 0.331 & - & 5.7437 & 0.318 & & - \\
\hline 14 & 0.296 & - & 5.6559 & 0.286 & & 0.342 \\
\hline
\end{tabular}


Firstly, we can get the original sequence data of trust vector $X^{(0)}(k)=\{0.993,0.862,0.633, \ldots, 0.342,0.358\}$. After performing the natural logarithm operations on the original data sequence, the sequence is modified to $X^{(0)}(k)=\{6.9007$, $6.7593,6.4504, \ldots, 5.8348,5.8805\}$. We can obtain, $a=-0.015417364, b=-448.2691709$, and the specific equation,

$$
\hat{x}^{(0)}(k)=e^{a(k-1)} \frac{2 b\left(1-e^{-a}\right)}{1+e^{-a}}=6.91099192 e^{-0.015417364(k-1)} \quad k=1,2, \ldots n
$$

Then we can calculate the cluster centers or benchmarks via utilizing the fuzzy clustering method described in Section 7.2.2, and finally divide the relative errors into four clustering groups, shown in Table 6.

Table 6 The cluster center of each state \%

\begin{tabular}{|c|c|c|c|c|}
\hline State & $\boldsymbol{E}_{\boldsymbol{1}}$ & $\boldsymbol{E}_{\mathbf{2}}$ & $\boldsymbol{E}_{3}$ & $\boldsymbol{E}_{\mathbf{4}}$ \\
\hline Cluster Center (Benchmark) & $-29 \% \sim-10 \%$ & $-10 \% \sim-1 \%$ & $-1 \% \sim 6 \%$ & $6 \% \sim 13 \%$ \\
\hline
\end{tabular}

According to scope of the above four cluster centers, we can determine the state for each relative error shown in the following Table 7.

Table 7 State of each relative error

\begin{tabular}{|c|c|c|c|c|c|c|}
\hline Sequence No. & 1 & 2 & 3 & 4 & 5 & 6 \\
\hline Relative Error \% & -1.1292 & -4.7564 & -28.5941 & -14.1304 & 9.5368 & 6.5319 \\
\hline State & $E_{2}$ & $E_{2}$ & $E_{1}$ & $E_{1}$ & $E_{4}$ & $E_{4}$ \\
\hline Sequence No. & 7 & 8 & 9 & 10 & 11 & 12 \\
\hline Relative Error \% & 3.0249 & 6.9549 & -2.2728 & 2.3810 & -9.3567 & 4.4693 \\
\hline State & $E_{3}$ & $E_{4}$ & $E_{2}$ & $E_{3}$ & $E_{2}$ & $E_{3}$ \\
\hline
\end{tabular}

Basing on the above steps, the state probability distribution for the 13-th data is:

$$
P(1)=P_{0}\left(N_{3}\right) P_{3}^{(N)}(0) P_{3}^{(N)}=(0,0,1,0)\left[\begin{array}{cccc}
1 / 2 & 0 & 0 & 1 / 2 \\
1 / 4 & 1 / 4 & 1 / 2 & 0 \\
0 & 1 / 2 & 0 & 1 / 2 \\
0 & 1 / 3 & 1 / 3 & 1 / 3
\end{array}\right]=(0,1 / 2,0,1 / 2) \text {, we can get the prediction value } 0.324 \text {. }
$$

By using the one step transition probability matrix, we can get the state of the 14 prediction result:

$P(1)=P_{1}\left(N_{2}\right) P_{2}^{(N)}(1) P_{2}^{(N)}+P_{1}\left(N_{4}\right) P_{4}^{(N)}(1) P_{4}^{(N)}=(1 / 6,1 / 6,1 / 2,1 / 6)$, and the prediction value is 0.293 .

The experimental results indicate that, the new proposed trust prediction model has a higher precision. And the system cloud grey model is suitable in dealing with the trust prediction issue.

\section{Conclusion and Future Work}

Mobile ad hoc networks have attracted much interest in the research community due to their potential applications. However, the inherent characteristics of such networks make them vulnerable to a wide variety of attacks. The security concerned in these wireless networks remains a serious impediment to widespread adoption. In this paper, we focus on the security of routing protocol in MANETs. Firstly, we abstract a decentralized trust inference model. Then by extending the standard Ad hoc On-demand Multi-path Distance Vector protocol (AOMDV), we propose a novel light-weight trust-enhanced routing protocol combined with the trust model, named as TeAOMDV. The persuasive experiments have been conducted to simulate and present the effectiveness of this new protocol.

For future works, we plan to do a deep research on trust prediction mechanism, considering the deployment region, network applications and level of security required. Moreover, according to the mobility, channel bandwidth, link load, resource constraints and other constraints, a context-aware scheme is our next step where trust attributes and weight vector determination can be adjusted automatically.

\section{ACKOWNLEGEMENT}

We would like to thank anonymous referees for their helpful suggestions to improve this paper.

This research is sponsored by the Natural Science Foundation of China (NSFC) under Grant Nos. 61402245, 61572267 and 61272425, the Project funded by China Postdoctoral Science Foundation under Grand No. 2015T80696 and 2014M551870, the Shandong Provincial Natural Science Foundation No. ZR2014FQ010, the Qingdao Postdoctoral Application Research Funded Project, the Open Project Foundation of Shandong Provincial Key Laboratory of Software Engineering under Grant No. 2013SE01, PAPD, CICAEET and Foundation of Huawei under Grant No.YB2013120027. And it is partially supported by NSF CNS-1015802, HK GRF 123609, China Thousand-Talent Program, China National 863 Program 2013AA013202.

\section{REFERENCES}

[1] Govindan, K.; Mohapatra, P.. (2012) Trust Computations and Trust Dynamics in Mobile Adhoc Networks: A Survey. IEEE Communications Surveys and Tutorials, 14(2), 279-298. 
[2] Li, W.; Parker, J.; Joshi, A.. (2012) Security through Collaboration and Trust in MANETs. Mobile Networks \& Applications, 17(3), 342-352.

[3] Wang, Jian.; Liu, Y.; Jiao Y. (2011) Building a trusted route in a mobile ad hoc network considering communication reliability and path length. Journal of Network and Computer Applications, 34(4), 1138-1149.

[4] Liang Z.; Shi W.. (2008) Analysis of ratings on trust inference in open environments. Performance Evaluation, 65(2), 99-128.

[5] Xia, H.; Jia, Z.; Li, X.; Ju L. and Sha. E.. (2013) Impact of Trust Model on On-demand Multi-path Routing in Mobile Ad Hoc Networks. Computer Communications, 36(9), 1078-1093.

[6] Velloso, P.; Laufer, R.; Cunha, D.; Duarte, O. and Pujolle, G.. (2010) Trust management in mobile ad hoc networks using a scalable maturity-based model. IEEE Transactions on Network and Service Management, 7(3), 172-185.

[7] Cho, J.; Swami, A.; Chen, I.. (2011) A Survey on Trust Management for Mobile Ad Hoc Networks. IEEE Communications Surveys and Tutorials, 13(4), 562-583.

[8] Cho, J.; Chen, I.. (2013) On the tradeoff between altruism and selfishness in MANET trust management. Ad Hoc Networks, 11(8), 2217-2234.

[9] Carullo, G.; Castiglione, A.; Cattaneo, G.; De Santis, A.; Fiore, U.; Palmieri, F.. (2013) FeelTrust: Providing Trustworthy Communications in Ubiquitous Mobile Environment. IEEE 27th International Conference on Advanced Information Networking and Applications (AINA), 1113-1120.

[10] Elgohary, Ashraf; Sobh, Tarek S.; Nouh, Sayed A.; Zaki, M.. (2014) An efficient and dependable protocol for critical MANETs. Journal of High Speed Networks, 20 (3), 153-168.

[11] Onolaja O.; Bahsoon, R.; Theodoropoulos, G.. (2011) Trust dynamics: a data-driven simulation approach. Trust Management V, 358, 323-334.

[12] Xia,H.; Jia, Z.; Li, X.; Ju, L. and Sha, E.. (2013) Trust prediction and trust-based source routing in mobile ad hoc networks. Ad Hoc Networks, 11(7), 2096-2114.

[13] Deb, N.; Chaki, N.. (2012) TIDS: Trust-based intrusion detection system for wireless ad-hoc networks. Computer Information Systems and Industrial Management, 7564, 80-91.

[14] Marchang, N.; Datta, R.. (2012) Light-weight trust-based routing protocol for mobile ad hoc networks. IET-Information Security, 6(2), 77-83.

[15]Eissa, T.; Razak, S; Khokhar, R.. (2013) Trust-Based Routing Mechanism in MANET: Design and Implementation. Mobile Networks \& Applications, 18(5), 666-677.

[16] Mohanapriya, M.; Krishnamurthi, I.. (2014) Trust based DSR routing protocol for mitigating cooperative black hole attacks in ad hoc networks. Arabian Journal for Science and Engineering, 39(3), 1825-1833.

[17] Rahimi, M.; Riazi, A. (2014) On local entropy of fuzzy partitions. Fuzzy Sets and Systems, 234, 97-108.

[18] Krishnendu Shaw; Ravi Shankar; Surendra S. Yadav; Lakshman S. Thakur. (2012) Supplier selection using fuzzy AHP and fuzzy multi-objective linear programming for developing low carbon supply chain. Expert Systems with Applications, 39(9), 8182-8192.

[19] http://www.isi.edu/nsnam/ns/, accessed December, 2012.

[20] Shen, J.; Tan, H.; Wang, J.; Wang, J. and Lee, S.. (2015) A Novel Routing Protocol Providing Good Transmission Reliability in Underwater Sensor Networks. Journal of Internet Technology, 16(1), 171-178.

[21] Chen, I.R.; Bao, F.Y.; Chang, M.; Cho, J.H.. (2014) Dynamic Trust Management for Delay Tolerant Networks and Its Application to Secure Routing. IEEE Transactions on Parallel and Distributed Systems, 25(5), 1200-1210.

[22]Zhao, H.Y.; Yang, X.; Li, X.L.. (2013) cTrust: Trust Management in Cyclic Mobile Ad Hoc Networks. IEEE Transactions on Vehicular Technology, 62(6), 2792-2806.

[23] Wu, Gin-Der; Zhu, Zhen-Wei. (2014) An enhanced discriminability recurrent fuzzy neural network for temporal classification problems. Fuzzy Sets and Systems, 237, 47-62.

[24]Zhang, L.X.; Lam, J. (2010) Necessary and sufficient conditions for analysis and synthesis of Markov jump linear systems with incomplete transition descriptions. IEEE Transactions on Automatic Control, 55(7), 1695-1701.

[25] Xie, Y.H.; Hu, J.K.; Xiang, Y.H.; Yu, S.; Tang, S.S.; Wang, Y.. (2013) Modeling Oscillation Behavior of Network Traffic by Nested Hidden Markov Model with Variable State-Duration. IEEE Transactions on Parallel and Distributed Systems, 24(9), 1807-1817.

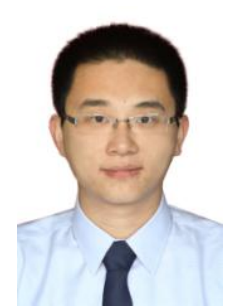

Dr. Hui Xia, born in 1986, is currently a Lecture in the College of Information Engineering at Qingdao University, China. His research interests focus on network and information security, trust computing, mobile computing, embedded system and cryptology. His research is sponsored by the Natural Science Foundation of China (NSFC) under Grant Nos. 61402245, the Project funded by China Postdoctoral Science Foundation under Grand No. 2014M551870, the Qingdao Postdoctoral Application Research Funded Project, the Open Project Foundation of Shandong Provincial Key Laboratory of Software Engineering under Grant No. 2013SE01. (xiahui@sdu.edu.cn)

Dr. Jia Yu, born in 1976, is currently a Professor in the College of Information Engineering at Qingdao

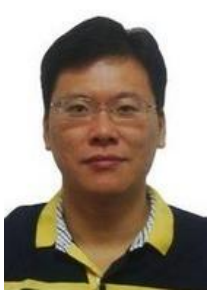
University, China. His main research interests include cryptology, network security, cloud security, and data security. (qduyujia@gmail.com) 


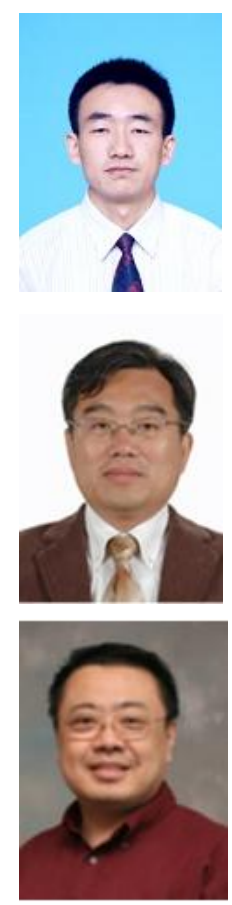

Dr. Cheng-liang Tian, born in 1983, is currently a Lecture in the College of Information Engineering at Qingdao University, China. His current research interest is lattice-based cryptography, network and information security. (tianchengliang@qdu.edu.cn)

Dr. Zhen-kuan Pan, born in 1966, is currently a Professor and Ph.D. supervisor in the College of Information Engineering at Qingdao University, China. His main research interests include virtual reality technology, computer vision, image science, and network and information security. (zkpan@qdu.edu.cn) Junior Faculty Enhancement Award, Notre Dame CSE Teaching Award, NSF CAREER Award, NSF ITR grant, Microsoft Trustworthy Curriculum Award, NSFC Distinguished Overseas Young Scholar, and ChangJiang Scholar Honorary Chair Professorship. His research has been supported by NSF (CAREER, ITR, EIA, IIS, CNS), Texas Instruments, AT \\&T, Texas Advanced Research Program, Microsoft, USA, etc. He is a member of ACM and IEEE, and is a senior member of China Computer Federation. (edsha@utdallas.edu) 